\title{
The Marcinkiewicz multiplier condition for bilinear operators
}

\author{
by \\ Loukas Grafakos and Nigel J. Kalton (Columbia, MO)
}

\begin{abstract}
This article is concerned with the question of whether Marcinkiewicz multipliers on $\mathbb{R}^{2 n}$ give rise to bilinear multipliers on $\mathbb{R}^{n} \times \mathbb{R}^{n}$. We show that this is not always the case. Moreover, we find necessary and sufficient conditions for such bilinear multipliers to be bounded. These conditions in particular imply that a slight logarithmic modification of the Marcinkiewicz condition gives multipliers for which the corresponding bilinear operators are bounded on products of Lebesgue and Hardy spaces.
\end{abstract}

1. Introduction. In this article we study bilinear multipliers of Marcinkiewicz type. Recall that a function $\sigma(\xi, \eta)=\sigma\left(\xi_{1}, \ldots, \xi_{n}, \eta_{1}, \ldots, \eta_{n}\right)$ defined away from the coordinate axes on $\mathbb{R}^{2 n}$, which satisfies the conditions

$$
\left|\partial_{\xi}^{\alpha} \partial_{\eta}^{\beta} \sigma(\xi, \eta)\right| \leq C_{\alpha, \beta}\left|\xi_{1}\right|^{-\alpha_{1}} \ldots\left|\xi_{n}\right|^{-\alpha_{n}}\left|\eta_{1}\right|^{-\beta_{1}} \ldots\left|\eta_{n}\right|^{-\beta_{n}}
$$

for sufficiently large multi-indices $\alpha=\left(\alpha_{1}, \ldots, \alpha_{n}\right)$ and $\beta=\left(\beta_{1}, \ldots, \beta_{n}\right)$, is called a Marcinkiewicz multiplier. It is a classical result (see for instance [18]) that Marcinkiewicz multipliers give rise to bounded linear operators $M_{\sigma}$ from $L_{p}\left(\mathbb{R}^{2 n}\right)$ into itself for $1<p<\infty$. Here $M_{\sigma}$ is the multiplier operator with symbol $\sigma$, that is,

$$
M_{\sigma}(F)(x)=\int_{\mathbb{R}^{2 n}} \widehat{F}(\zeta) \sigma(\zeta) e^{2 \pi i\langle x, \zeta\rangle} d \zeta
$$

where $F$ is a Schwartz function on $\mathbb{R}^{2 n}$ and $\widehat{F}(\zeta)$ is the Fourier transform of $F$, defined by $\widehat{F}(\zeta)=\int_{\mathbb{R}^{2 n}} F(x) e^{-2 \pi i\langle x, \zeta\rangle} d x$. (We will use the notation $\langle x, y\rangle=\sum_{k=1}^{m} x_{k} y_{k}$ for $x=\left(x_{1}, \ldots, x_{m}\right)$ and $y=\left(y_{1}, \ldots, y_{m}\right)$ elements of $\mathbb{R}^{m}$.) The Marcinkiewicz condition (1.1) is less restrictive than the Hörmander-Mikhlin condition

$$
\left|\partial_{\xi}^{\alpha} \partial_{\eta}^{\beta} \sigma(\xi, \eta)\right| \leq C_{\alpha, \beta}(|\xi|+|\eta|)^{-|\alpha|-|\beta|},
$$

2000 Mathematics Subject Classification: Primary 42B15, 42B20, 42B30; Secondary 46B70, 47G30.

Key words and phrases: Marcinkiewicz condition, bilinear multipliers, paraproducts.

The research of both authors was partially supported by the NSF. 
which is also known to imply boundedness for the linear operator $W_{\sigma}$ from $L_{p}\left(\mathbb{R}^{2 n}\right)$ into itself when $1<p<\infty$. The advantage of condition (1.2) is that it is supposed to hold for multi-indices up to order $|\alpha|+|\beta| \leq n+1$ versus up to order $|\alpha|+|\beta| \leq 2 n$ for condition (1.1).

In this paper we study bilinear multiplier operators whose symbols satisfy similar conditions. More precisely, we are interested in boundedness properties of bilinear operators

$$
W_{\sigma}(f, g)(x)=\int_{\mathbb{R}^{2 n}} \widehat{f}(\xi) \widehat{g}(\eta) \sigma(\xi, \eta) e^{2 \pi i\langle x, \xi\rangle} e^{2 \pi i\langle x, \eta\rangle} d \xi d \eta,
$$

originally defined for $f, g$ Schwartz functions on $\mathbb{R}^{n}$ and $\sigma$ a function on $\mathbb{R}^{2 n}$. A well known theorem of Coifman and Meyer [4] says that if the function $\sigma$ on $\mathbb{R}^{2 n}$ satisfies (1.2) for sufficiently large multi-indices $\alpha$ and $\beta$, then the bilinear map $W_{\sigma}(f, g)$ extends to a bounded operator from $L_{p_{1}}\left(\mathbb{R}^{n}\right) \times$ $L_{p_{2}}\left(\mathbb{R}^{n}\right)$ into $L_{p_{0}, \infty}\left(\mathbb{R}^{n}\right)$ when $1<p_{1}, p_{2}<\infty, 1 / p_{1}+1 / p_{2}=1 / p_{0}$ and $p_{0} \geq 1$. (Here $L_{p_{0}, \infty}$ denotes the space weak $L_{p_{0}}$.) This result was later extended to the range $1>p_{0} \geq 1 / 2$ by Grafakos and Torres [9] and Kenig and Stein [11]. The extension into $L_{p_{0}}$ for $p_{0}<1$ was stimulated by the recent work of Lacey and Thiele [12] who showed that the discontinuous symbol $\sigma(\xi, \eta)=-i \operatorname{sgn}(\xi-\eta)$ on $\mathbb{R}^{2}$ gives rise to a bounded bilinear operator $W_{\sigma}$ from $L_{p_{1}}(\mathbb{R}) \times L_{p_{2}}(\mathbb{R})$ into $L_{p_{0}}(\mathbb{R})$ for $2 / 3<p_{0}<\infty$ when $1<p_{1}, p_{2}<$ $\infty$ and $1 / p_{1}+1 / p_{2}=1 / p_{0}$.

In this article we address the question of whether the Marcinkiewicz condition (1.1) on $\mathbb{R}^{2 n}$ gives rise to a bounded bilinear operator $W_{\sigma}$ on $\mathbb{R}^{n} \times \mathbb{R}^{n}$. We answer this question negatively. More precisely, we show that there exist examples of bounded functions $\sigma(\xi, \eta)$ on $\mathbb{R}^{n} \times \mathbb{R}^{n}$ which satisfy the stronger condition

$$
\left|\partial_{\xi}^{\alpha} \partial_{\eta}^{\beta} \sigma(\xi, \eta)\right| \leq C_{\alpha, \beta}|\xi|^{-|\alpha|}|\eta|^{-|\beta|}
$$

for all multi-indices $\alpha$ and $\beta$, for which the corresponding bilinear operators $W_{\sigma}$ do not map $L_{p_{1}} \times L_{p_{2}}$ into $L_{p_{0}, \infty}$ for any triple of exponents satisfying $1 / p_{1}+1 / p_{2}=1 / p_{0}$ and $1<p_{1}, p_{2}<\infty$.

We reduce this problem to the study of bilinear operators of the type

$$
(f, g) \mapsto \sum_{j \in \mathbb{Z}} \sum_{k \in \mathbb{Z}} a_{j k} \widetilde{\Delta}_{j} f \widetilde{\Delta}_{k} g,
$$

where $a_{j k}$ is a bounded sequence of scalars depending on $\sigma$ and $\widetilde{\Delta}_{j}$ are the Littlewood-Paley operators given by multiplication on the Fourier transform side by a smooth bump supported near the frequency $|\xi| \sim 2^{j}$. In Section 6 , in particular Theorem 6.5, we find a necessary and sufficient condition on the infinite matrix $A=\left(a_{j k}\right)_{j, k}$ so that the bilinear operator in (1.4) maps $L_{p_{1}} \times L_{p_{2}}$ into $L_{p_{0}, \infty}$. This condition is expressed in terms of an Orlicz space 
norm of the sequence $\left(a_{j k}\right)_{j, k}$. It turns out that this condition is independent of the exponents $p_{1}, p_{2}, p_{0}$ and depends only on quantities intrinsic to the matrix $A$ (although the actual norm of the operator in (1.4) from $L_{p_{1}} \times L_{p_{2}}$ into $L_{p_{0}, \infty}$ does depend on the indices $\left.p_{1}, p_{2}, p_{0}\right)$.

The results of Section 6 are transferred in Section 7 to multiplier theorems for bilinear operators. This transference is achieved by using a Fourier expansion of the symbol $\sigma$ on products of dyadic cubes. Theorem 7.2 is the main result of that section and Theorem 7.3 shows that this theorem is best possible. Theorem 7.2 allows us to derive that the estimates

$$
\left|\partial_{\xi}^{\alpha} \partial_{\eta}^{\beta} \sigma(\xi, \eta)\right| \leq C_{\alpha, \beta}|\xi|^{-|\alpha|}|\eta|^{-|\beta|}\left(\log \left(1+\left|\log \frac{|\xi|}{|\eta|}\right|\right)\right)^{-\theta}
$$

do give rise to a bounded bilinear operator $W_{\sigma}$ on products of $L_{p}$ spaces when $\theta>1$, while we show that this is not the case when $0<\theta<1 / 2$. We obtain similar results when the expression $(\log (1+|\log (|\xi| /|\eta|)|))^{-\theta}$ in (1.5) is replaced by the expression

$$
\left(\log \left(1+\left|\log \frac{|\xi|}{|\eta|}\right|\right)\right)^{-1}\left(\log \left(1+\log \left(1+\left|\log \frac{|\xi|}{|\eta|}\right|\right)\right)\right)^{-\theta} \quad \text { for } \theta>1 .
$$

We find it more convenient to work with the martingale difference operators $\Delta_{k}$ associated with the $\sigma$-algebra of all dyadic cubes of size $2^{k}$ in $\mathbb{R}^{n}$ and later transfer our results to the Littlewood-Paley operators $\widetilde{\Delta}_{k}$. This point of view is introduced in the next section.

We end this article with a short discussion on paraproducts (see Section 8). These are operators of the type (1.4) for specific sequences $\left(a_{j k}\right)_{j, k}$ of zeros and ones.

2. A maximal operator. Let $(\Omega, \Sigma, \mathbb{P})$ be any probability space and let $\left(\Sigma_{k}\right)_{k>0}$ be a filtration, i.e. an increasing sequence of sub- $\sigma$-algebras of $\Sigma$. We say that $\left(\Sigma_{k}\right)$ is a dyadic filtration if each $\Sigma_{k}$ is atomic and has precisely $2^{k}$ atoms each with probability $2^{-k}$. We say $\left(\Sigma_{k}\right)$ is a $2^{n}$-adic filtration if each $\Sigma_{k}$ is atomic with precisely $2^{n k}$ atoms each with probability $2^{-n k}$.

Associated with $\Sigma_{k}$ we define the conditional expectation operators $\mathcal{E}_{k} f$ $=\mathbb{E}\left(f \mid \Sigma_{k}\right)$ and the martingale difference operators $\Delta_{k} f=\mathcal{E}_{k} f-\mathcal{E}_{k-1} f$ for $k \geq 1$ and $f \in L_{1}(\Omega)$.

Let $A=\left(a_{j k}\right)$ be a complex $M \times N$ matrix, and let $(\Omega, \Sigma, \mathbb{P})$ be a probability space with a dyadic filtration $\left(\Sigma_{k}\right)_{k \geq 0}$. For $1 \leq p<\infty$ we define $h_{p}(A)$ to be the least constant so that for all $f \in L_{p}(\Omega)$ we have

$$
\left\|\max _{1 \leq j \leq M}\left|\sum_{k=1}^{N} a_{j k} \Delta_{k} f\right|\right\|_{L_{p}} \leq h_{p}(A)\|f\|_{L_{p}} .
$$


We also define the corresponding weak constants, i.e. the least constants so that for all $f \in L_{p}(\Omega)$ we have

$$
\left\|\max _{1 \leq j \leq M}\left|\sum_{k=1}^{N} a_{j k} \Delta_{k} f\right|\right\|_{L_{p, \infty}} \leq h_{p}^{\mathrm{w}}(A)\|f\|_{L_{p}} .
$$

Finally, for $0<q<p<\infty$ we define the mixed constants $h_{p, q}(A)$ as the least constants such that for all $f \in L_{p}(\Omega)$ we have

$$
\left\|\max _{1 \leq j \leq M}\left|\sum_{k=1}^{N} a_{j k} \Delta_{k} f\right|\right\|_{L_{q}} \leq h_{p, q}(A)\|f\|_{L_{p}} .
$$

Note that these definitions are independent of the choice of the probability space and of the dyadic filtration. Indeed, if $A$ is fixed, it suffices to take $f \in L_{p}\left(\Sigma_{N}\right)$ and hence we can consider a finite probability space with $2^{N}$ points and a finite dyadic filtration $\left(\Sigma_{k}\right)_{k=0}^{N}$. We also note that $h_{p}(A)$ is the operator norm of the map $T_{A}: L_{p}(\Omega) \rightarrow L_{p}\left(\Omega ; \ell_{\infty}^{M}\right)$ defined by

$$
T_{A} f=\left(\sum_{k=1}^{N} a_{j k} \Delta_{k} f\right)_{j=1}^{M} .
$$

Similarly, $h_{p}^{\mathrm{w}}(A)$ is the norm of the operator $T_{A}: L_{p} \rightarrow L_{p, \infty}\left(\Omega ; \ell_{\infty}^{M}\right)$.

Our first result is that all these constants are mutually equivalent, when $1<p<\infty$ :

TheOREm 2.1. If $1<p, q<\infty$ then there is a constant $0<C=$ $C(p, q)<\infty$ such that for all complex $M \times N$ matrices $A$ we have

$$
\frac{1}{C} h_{p}(A) \leq h_{q}^{\mathrm{w}}(A) \leq h_{q}(A) \leq C h_{p}(A) .
$$

Proof. It suffices to prove an estimate of the type $h_{p}(A) \leq C h_{q}^{\mathrm{w}}(A)$ for any choice of $1<p, q<\infty$. We first prove a weak type $(1,1)$ estimate for $T_{A}$, i.e. that $h_{1}^{\mathrm{w}}(A) \leq C h_{q}^{\mathrm{w}}(A)$. Suppose $f \in L_{1}$ with $\|f\|_{L_{1}}=1$. Then if $\lambda, \gamma>0$, with $\lambda \gamma>1$, we can use an appropriate Calderón-Zygmund decomposition to find finite sets $D_{1}, \ldots, D_{m}$ so that each $D_{l}$ is an atom of some $\Sigma_{l}$,

$$
\gamma \lambda \leq \mathbb{P}\left(D_{l}\right)^{-1} \int_{D_{l}}|f| d \mathbb{P}=\underset{D_{l}}{\operatorname{Ave}} f \leq 2 \gamma \lambda,
$$

and $|f(\omega)| \leq \gamma \lambda$ if $\omega \notin \bigcup_{l=1}^{m} D_{l}$. Let

$$
g=\sum_{l=1}^{m}\left(\underset{D_{l}}{\operatorname{Ave} f}\right) \chi_{D_{l}}
$$

and $E=\bigcup_{l=1}^{m} D_{l}$. Then $T_{A}\left(f \chi_{E}-g\right)$ is supported in $E$ and thus

$$
\mathbb{P}\left(\left\|T_{A}\left(f \chi_{E}-g\right)\right\|_{\ell_{\infty}^{M}}>\lambda / 2\right) \leq \mathbb{P}(E) \leq(\gamma \lambda)^{-1} .
$$


On the other hand, $\left\|f-f \chi_{E}+g\right\|_{L_{\infty},} \leq 3 \gamma \lambda$ and $\left\|f-f \chi_{E}+g\right\|_{L_{1}} \leq 1$. Hence $\left\|f-f \chi_{E}+g\right\|_{L_{q}} \leq 3^{1 / q^{\prime}}(\gamma \lambda)^{1 / q^{\prime}}$ and so

$$
\left\|T_{A}\left(f-f \chi_{E}+g\right)\right\|_{L_{q, \infty}\left(\ell_{\infty}^{M}\right)} \leq h_{q}^{\mathrm{w}}(A) 3^{1 / q^{\prime}}(\gamma \lambda)^{1 / q^{\prime}},
$$

which implies that

$$
\mathbb{P}\left(\left\|T_{A}\left(f-f \chi_{E}+g\right)\right\|_{\ell_{\infty}^{M}}>\lambda / 2\right) \leq \frac{2^{q}}{\lambda^{q}}\left(h_{q}^{\mathrm{w}}(A)\right)^{q} 3^{q-1}(\gamma \lambda)^{q-1} .
$$

Selecting $\gamma=1 / h_{q}^{\mathrm{w}}(A)$ and combining with (2.4) we obtain (for $\lambda>h_{q}^{\mathrm{w}}(A)$ )

$$
\lambda \mathbb{P}\left(\left\|T_{A} f\right\|_{\ell_{\infty}^{M}}>\lambda\right) \leq C h_{q}^{\mathrm{w}}(A)
$$

where $C=C(p, q)$. This gives the weak-type $(1,1)$ estimate for $T_{A}$. Now by the Marcinkiewicz interpolation theorem (applied to the sublinear map $f \mapsto$ $\left.\left\|T_{A} f(\omega)\right\|_{\ell_{\infty}^{M}}\right)$ we deduce that $h_{p}(A) \leq C(p, q) h_{q}^{\mathrm{w}}(A)$ as long as $1<p<q$.

We now prove that $h_{p}(A) \leq C(p, q) h_{q}^{\mathrm{w}}(A)$ when $1<q<p<\infty$. We consider the dual map $T_{A}^{*}: L_{1}\left(\Omega ; \ell_{1}^{M}\right) \rightarrow L_{1}$ defined by

$$
T_{A}^{*} \mathbf{f}=\sum_{j=1}^{M} \sum_{k=1}^{N} a_{j k} \Delta_{k} f_{j}
$$

where $\mathbf{f}(\omega)=\left(f_{j}(\omega)\right)_{j=1}^{M}$. We find that $T_{A}^{*}: L_{r}\left(\Omega ; \ell_{1}^{M}\right) \rightarrow L_{r}$ has norm bounded by $C(q, r) h_{q}^{\mathrm{w}}(A)$ as long as $1<r^{\prime}<q$, i.e. $q^{\prime}<r<\infty$. Using this $r$ as a starting point, we repeat the argument above to show that $T_{A}^{*}: L_{1}\left(\Omega ; \ell_{1}^{M}\right) \rightarrow L_{1, \infty}$ has norm bounded by $C h_{q}^{\mathrm{w}}(A)$. The Marcinkiewicz interpolation theorem can again be used to show that $T_{A}^{*}: L_{p^{\prime}}\left(\Omega, \ell_{1}^{M}\right) \rightarrow L_{p^{\prime}}$ has norm bounded by $C h_{q}^{\mathrm{w}}(A)$ for all $1<p^{\prime}<r$, and thus in particular when $1<p^{\prime}<q^{\prime}$. Therefore we conclude that $h_{p}(A) \leq C h_{q}^{\mathrm{w}}(A)$ when $1<q<p<\infty$.

Remark. From now we will write $h(A)=h_{2}(A)$ so that each $h_{p}(A)$ for $1<p<\infty$ is equivalent to $h(A)$.

It is of some interest to observe that even the corresponding mixed constants are also equivalent to $h(A)$.

Theorem 2.2. Suppose $0<q<p$ and $1<p<\infty$. Then there is a constant $C=C(p, q)$ so that

$$
\frac{1}{C} h(A) \leq h_{p, q}(A) \leq C h(A)
$$

Proof. This will depend on the following lemma:

Lemma 2.3. Suppose $1 \leq p<\infty$ and $0<q<p$. Then there is a constant $C=C(p, q)$ so that if $r=\min (p, 2)$ we have

$$
\left\|T_{A}\right\|_{L_{p} \rightarrow L_{r, \infty}\left(\ell_{\infty}^{M}\right)} \leq C h_{p, q}(A) .
$$


Proof of Lemma 2.3. We may assume $q<r$. The proof is a fairly standard application of Nikishin's theorem (see [16]). Here we use a version given in [17]. It is simplest to consider the case when $\Omega$ is finite with $|\Omega|=2^{N}$. Consider the map $T_{A}: L_{p} \rightarrow L_{q}\left(\Omega ; \ell_{\infty}^{M}\right)$. For each $f \in L_{p}$ with $\|f\|_{L_{p}} \leq 1$, let $F_{f}(x)=\left\|T_{A} f(x)\right\|_{\ell_{\infty}^{M}}$. For $\left\|f_{j}\right\|_{L_{p}} \leq 1$ with $1 \leq j \leq J, \sum_{j=1}^{J}\left|b_{j}\right|^{r}=1$, and $\left(\varepsilon_{j}\right)_{j=1}^{J}$ a sequence of independent Bernoulli random variables on some probability space, we have

$$
\left\|\max _{1 \leq j \leq J}\left|b_{j}\right| F_{f_{j}}\right\|_{L_{q}} \leq \mathbb{E}\left(\left\|\sum_{j=1}^{J} \varepsilon_{j} b_{j} T_{A} f_{j}\right\|_{L_{q}\left(\ell_{\infty}^{M}\right)}\right) \leq C h_{p, q}(A),
$$

since $L_{p}$ has type $r$. It follows from [17] that there is a function $w \in L_{1}$, with $\int w d \mathbb{P}=1$, and $w \geq 0$ a.e., such that for any set $E \subset \Omega$,

$$
\left(\int_{E} F_{f}^{q} d \mathbb{P}\right)^{1 / q} \leq C h_{p, q}(A)\left(\int_{E} w d \mathbb{P}\right)^{1 / q-1 / r} .
$$

Now consider the set $S$ of all permutations of $\Omega$ which induce permutations of the atoms of each $\Sigma_{k}$ for $1 \leq k \leq N$; there are $2^{2^{N}-1}$ such permutations $\varphi$. For $\varphi \in S$ we have

$$
\left(\int_{E} F_{f \circ \varphi}^{q} d \mathbb{P}\right)^{1 / q} \leq C h_{p, q}(A)\left(\int_{E} w d \mathbb{P}\right)^{1 / q-1 / r}
$$

or equivalently

$$
\left(\int_{E} F_{f}^{q} d \mathbb{P}\right)^{1 / q} \leq C h_{p, q}(A)\left(\int_{E} w \circ \varphi^{-1} d \mathbb{P}\right)^{1 / q-1 / r} .
$$

Raising to the power $(1 / q-1 / r)^{-1}$, averaging over $S$, and then raising to the power $1 / q-1 / r$ gives

$$
\left(\int_{E} F_{f}^{q} d \mathbb{P}\right)^{1 / q} \leq C h_{p, q}(A)\left(\frac{1}{|S|} \sum_{\varphi \in S}\left(\int_{E} w \circ \varphi^{-1} d \mathbb{P}\right)\right)^{1 / q-1 / r} .
$$

But this implies

$$
\left(\int_{E} F_{f}^{q} d \mathbb{P}\right)^{1 / q} \leq C h_{p, q}(A) \mathbb{P}(E)^{1 / q-1 / r},
$$

which gives the required weak type estimate (2.8).

We now return to the proof of Theorem 2.2. We first observe that we always have $h_{p, q}(A) \leq C h_{p}^{\mathrm{w}}(A)$ since $q<p$. If $1<p \leq 2$, Lemma 2.3 gives $h_{p}^{\mathrm{w}}(A) \leq C h_{p, q}(A)$ and the required conclusion follows from Theorem 2.1. Assume therefore that $p>2$ and that $T_{A}$ maps $L_{p}$ into $L_{q}\left(\ell_{\infty}^{M}\right)$ with norm $h_{p, q}(A)$. Fix $f$ with $\|f\|_{L_{1}}=1$ and use the Calderón-Zygmund decomposition of Theorem 2.1 to obtain (2.4) as before, but instead of (2.5) the 
estimate

$$
\left\|T_{A}\left(f-f \chi_{E}+g\right)\right\|_{L_{q}} \leq h_{p, q}(A) 3^{1 / p^{\prime}}(\gamma \lambda)^{1 / p^{\prime}}
$$

which implies

$$
\mathbb{P}\left(\left\|T_{A}\left(f-f \chi_{E}+g\right)\right\|_{\ell_{\infty}^{M}}>\lambda / 2\right) \leq \frac{2^{q}}{\lambda^{q}}\left(h_{p, q}(A)\right)^{q} 3^{q / p^{\prime}}(\gamma \lambda)^{q / p^{\prime}} .
$$

Selecting $\gamma=h_{p, q}(A)^{-s} \lambda^{s-1}$ with $1 / s=1 / p^{\prime}+1 / q$ and combining with (2.4) we obtain

$$
\lambda \mathbb{P}\left(\left\|T_{A} f\right\|_{\ell_{\infty}^{M}}>\lambda\right)^{1 / s} \leq C h_{p, q}(A) .
$$

This says that $T_{A}$ maps $L_{1}$ into $L_{s, \infty}\left(\ell_{\infty}^{M}\right)$ with norm at most $C h_{p, q}(A)$, in particular that $T_{A}$ maps $L_{1}$ into $L_{t}\left(\ell_{\infty}^{M}\right)$ as long as $0<t<s$. Lemma 2.3 implies that $T_{A}$ maps $L_{p}$ into $L_{2, \infty}\left(\ell_{\infty}^{M}\right)$ and also $L_{1}$ into $L_{2, \infty}\left(\ell_{\infty}^{M}\right)$ with norms at most a multiple of $h_{p, q}(A)$. By interpolation it follows that $T_{A}$ maps $L_{r}$ into $L_{2, \infty}\left(\ell_{\infty}^{M}\right) \subset L_{r, \infty}\left(\ell_{\infty}^{M}\right)$ for $1 \leq r \leq 2$. We conclude that $h_{r}^{\mathrm{w}}(A) \leq C h_{p, q}(A)$ for $1<r<2$ but since $h_{r}^{\mathrm{w}}(A)$ is comparable to $h_{p}^{\mathrm{w}}(A)$, we finally obtain $h_{p}^{\mathrm{w}}(A) \leq C h_{p, q}(A)$. Since the converse inequality is always valid when $q<p$, we apply Theorem 2.1 to conclude the proof.

We next prove the elementary observation that, for $1<p<\infty, h(A)$ remains unchanged under interpolation of extra columns or extra rows of zeros.

Lemma 2.4. Let $A$ be a complex $M \times N$ matrix and $\left(m_{r}\right)_{r=1}^{M},\left(n_{s}\right)_{s=1}^{N}$ be two increasing finite sequences of natural numbers. Suppose $M_{1} \geq m_{M}$ and $N_{1} \geq n_{N}$. Let $B=\left(b_{j k}\right)$ be the $M_{1} \times N_{1}$ matrix defined by $b_{j k}=a_{r s}$ when $j=m_{r}$ and $k=n_{s}$, and $b_{j k}=0$ otherwise. Then $h(A)=h(B)$.

Proof. Interpolating extra rows of zeros is trivial, so we can assume $m_{r}=$ $m$ for all $r$. For the case of columns, we only need to show that $h(B) \leq h(A)$. We may suppose that $\Omega$ is a finite set with $2^{N_{1}}$ points and that $\left(\Sigma_{k}\right)_{k=0}^{N_{1}}$ is a finite dyadic filtration of $\Omega$. It is then possible to write $\Omega=\Omega_{1} \times \Omega_{2}$ where $\left|\Omega_{1}\right|=2^{N_{1}-N}$ and $\left|\Omega_{2}\right|=2^{N}$, and find a dyadic filtration $\left(\Sigma_{k}^{(1)}\right)_{k=0}^{N_{1}-N}$ of $\Omega_{1}$ and a dyadic filtration $\left(\Sigma_{k}^{(2)}\right)_{k=0}^{N}$ of $\Omega_{2}$ so that $\Sigma_{k}^{(1)} \times \Sigma_{k}^{(2)}=\Sigma_{n_{k}}$ for $0 \leq k \leq N$, and $\Sigma_{k+1}^{(1)} \times \Sigma_{k}^{(2)}=\Sigma_{n_{k+1}-1}$ for $0 \leq k \leq N-1$. Then for $f \in L_{2}\left(\Omega_{1} \times \Omega_{2}\right)$ let $g=\sum_{k=1}^{N} \Delta_{k} f$ and note that

$$
\Delta_{n_{k}} f\left(\omega_{1}, \omega_{2}\right)=\Delta_{k}^{(2)} g_{\omega_{1}}\left(\omega_{2}\right),
$$

where $g_{\omega_{1}}\left(\omega_{2}\right)=g\left(\omega_{1}, \omega_{2}\right)$. Hence

$$
\int_{\Omega_{2}} \sup _{j}\left|\sum_{k=1}^{N} a_{j, n_{k}} \Delta_{n_{k}} f\left(\omega_{1}, \omega_{2}\right)\right|^{2} d \omega_{2} \leq h_{p}(A) \int_{\Omega_{2}}\left|g\left(\omega_{1}, \omega_{2}\right)\right|^{2} d \omega_{2} .
$$


Integrating over $\Omega_{1}$ gives

$$
\left\|\sup _{j}\left|\sum_{k=1}^{N} a_{j, n_{k}} \Delta_{n_{k}} f\right|\right\|_{L_{2}} \leq h_{p}(A)\|g\|_{L_{2}} \leq h_{p}(A)\|f\|_{L_{2}} .
$$

We can now extend our definitions, replacing dyadic filtrations by $2^{n}$-adic filtrations:

Proposition 2.5. Suppose $n \in \mathbb{N}$ and $1<p<\infty$. Then there is a constant $C(p, n)$ with the following property. Let $(\Omega, \Sigma, \mathbb{P})$ be a probability space and suppose $\left(\Sigma_{k}\right)_{k=0}^{\infty}$ is a $2^{n}$-adic filtration. Let $A$ be any $M \times N$ matrix and let $h_{p}(A ; n)$ be the least constant so that

$$
\left\|\sup _{j}\left|\sum_{k=1}^{N} a_{j k} \Delta_{k} f\right|\right\|_{L_{p}} \leq h_{p}(A ; n)\|f\|_{L_{p}},
$$

and $h_{p}^{\mathrm{w}}(A ; n)$ be the least constant so that

$$
\left\|\sup _{j}\left|\sum_{k=1}^{N} a_{j k} \Delta_{k} f\right|\right\|_{L_{p, \infty}} \leq h_{p}^{\mathrm{w}}(A ; n)\|f\|_{L_{p}} .
$$

Then $h_{p}^{\mathrm{w}}(A) \leq h_{p}^{\mathrm{w}}(A ; n), h_{p}(A) \leq h_{p}(A ; n)$, and $h_{p}^{\mathrm{w}}(A ; n) \leq h_{p}(A ; n) \leq$ $C h(A)$.

Proof. This is essentially trivial; we need only prove that $h_{p}(A ; n) \leq$ $C h(A)$. To do this note that $h_{p}(A ; n)=h_{p}(B)$ where $B$ is obtained from $A$ by repeating each column $n$ times. The proposition then follows by the triangle law from Lemma 2.4.

3. Estimates for $h(A)$. We next turn to the problem of estimating $h(A)$. We shall assume that $(\Omega, \mathbb{P})$ is a fixed probability space with a dyadic filtration $\left(\Sigma_{k}\right)_{k=0}^{\infty}$. Our first estimate is trivial.

Proposition 3.1. There is a constant $C$ so that for any $M \times N$ matrix $A=\left(a_{j k}\right)$ we have

$$
h(A) \leq C \sup _{1 \leq j \leq M} \sum_{k=0}^{N}\left|a_{j k}-a_{j, k+1}\right|,
$$

where we set $a_{j 0}=a_{j, N+1}=0$ for all $1 \leq j \leq M$.

Proof. Suppose $f \in L_{2}$. Summation by parts gives

$$
\sum_{k=1}^{N} a_{j k} \Delta_{k} f=\sum_{k=0}^{N}\left(a_{j k}-a_{j, k+1}\right) \mathcal{E}_{k} f,
$$


thus

$$
\left|\sum_{k=1}^{N} a_{j k} \Delta_{k} f\right| \leq\left(\sup _{1 \leq j \leq M} \sum_{k=0}^{N}\left|a_{j k}-a_{j, k+1}\right|\right) \sup _{k}\left|\mathcal{E}_{k} f\right|,
$$

and the result follows because of the maximal estimate

$$
\left\|\sup _{k}\left|\mathcal{E}_{k} f\right|\right\|_{L_{2}} \leq C\|f\|_{L_{2}}
$$

proved in [8].

We next turn to the problem of getting a more delicate estimate. To do this we need the theory of a certain Lorentz space. Let $w=\left(w_{k}\right)_{k=1}^{\infty}$ be a decreasing sequence of positive numbers. We will consider the following two conditions on $w$ :

$$
\exists C>0, \exists \theta>0, \quad w_{k} \leq C\left(\frac{\log (j+1)}{\log (k+1)}\right)^{\theta} w_{j} \quad \text { when } 1 \leq j \leq k,
$$

(where throughout this paper log denotes the logarithm with base 2) and

$$
\sum_{k=1}^{\infty} \frac{w_{k}}{k}<\infty
$$

Roughly speaking, (3.1) means that $w_{k}$ decays logarithmically while (3.2) implies that it decays reasonably fast. Note $w_{k}=(\log (k+1))^{-\theta}$ satisfies $(3.1)$ if $\theta>0$ and (3.2) if $\theta>1$. The sequence $w_{k}=(\log (k+1))^{-1}(\log \log (k+2))^{-\theta}$ satisfies both (3.1) and (3.2) when $\theta>1$.

Now let $d=d(w, 1)$ be the Lorentz sequence space of all complex sequences $\mathbf{u}=\left(u_{k}\right)_{k \in \mathbb{Z}}$ such that

$$
\|\mathbf{u}\|_{d}=\sup _{\pi} \sum_{k \in \mathbb{Z}} w_{\pi(k)}\left|u_{k}\right|<\infty
$$

where the supremum is taken over all one-one maps $\pi: \mathbb{Z} \rightarrow \mathbb{N}$. The dual of $d(w, 1)$ can be naturally identified as the space $d^{*}=d^{*}(w, 1)$ consisting of all sequences $\left(v_{k}\right)_{k \in \mathbb{Z}}$ so that

$$
\sup _{k \in \mathbb{N}} \frac{v_{1}^{*}+\ldots+v_{k}^{*}}{w_{1}+\ldots+w_{k}}=\|\mathbf{v}\|_{d^{*}}<\infty
$$

where $\left(v_{k}^{*}\right)_{k=1}^{\infty}$ is the decreasing rearrangement of $\left(\left|v_{k}\right|\right)_{k \in \mathbb{Z}}$. We refer to [13], p. 175, for properties of Lorentz spaces. Note that under condition (3.1), $d(w, 1)$ is also an Orlicz sequence space (see [13], p. 176).

The following lemma is surely well known to specialists, but we include a proof anyway.

LEMMA 3.2. Under condition (3.1), the Lorentz space $d(w, 1)$ has cotype two. 
Proof. By combining Proposition 1.f.3 (p. 82) and Theorem 1.f.7 (p. 84) of [14] one sees that it is only necessary to show that $d(w, 1)$ has a lower $q$-estimate for some $q<2$. To do this observe that if $\mathbf{v}_{1}, \ldots, \mathbf{v}_{N}$ are disjointly supported sequences, then

$$
\left\|\sum_{j=1}^{N} \mathbf{v}_{j}\right\|_{d} \geq \inf _{k \geq 1} \frac{w_{k}}{w_{k N}} \sum_{j=1}^{N}\left\|\mathbf{v}_{j}\right\|_{d} .
$$

Hence

$$
\sum_{j=1}^{N}\left\|\mathbf{v}_{j}\right\|_{d(w, 1)} \leq C(\log (N+1))^{\theta}\left\|\sum_{j=1}^{N} \mathbf{v}_{j}\right\|_{d} .
$$

Now suppose $1<q<2$ and $\left\|\sum \mathbf{v}_{j}\right\|_{d}=1$. Then for each $s \in \mathbb{N}$, let $m_{s}$ be the number of $j$ so that $2^{-s}<\left\|\mathbf{v}_{k}\right\|_{d} \leq 2^{-s+1}$. Then

$$
m_{s} 2^{-s} \leq C\left(\log \left(m_{s}+1\right)\right)^{\theta} .
$$

This in turn implies that

$$
m_{s}^{1-\varrho} \leq C 2^{s}
$$

where $\varrho>0$ is chosen so that $(1-\varrho)^{-1}<q$. Then we obtain an estimate

$$
\sum_{j=1}^{N}\left\|\mathbf{v}_{j}\right\|_{d}^{q} \leq C \sum_{s=1}^{\infty} m_{s} 2^{-s q} \leq C^{\prime} .
$$

This establishes a lower $q$-estimate.

The norms $\|\cdot\|_{d}$ and $\|\cdot\|_{d^{*}}$ are of course defined for finite sequences with $M$ elements and thus can be thought of as norms on $\mathbb{C}^{M}$. We denote these spaces by $d(w, 1)^{(M)}$ and $d^{*}(w, 1)^{(M)}$.

Proposition 3.3. If $\left(w_{n}\right)$ satisfies both (3.1) and (3.2) then given $2<$ $p<\infty$ there is a constant $C$ so that for any sequence $\varepsilon_{k}= \pm 1$ and any $M, N \in \mathbb{N}$ we have the estimate

$$
\left(\mathbb{E}\left(\left\|\sum_{k=1}^{N} \varepsilon_{k} \Delta_{k} \mathbf{f}\right\|_{\ell_{\infty}}^{2}\right)\right)^{1 / 2} \leq C\left(\mathbb{E}\left(\|\mathbf{f}\|_{d^{*}}^{p}\right)\right)^{1 / p},
$$

for any $\mathbf{f} \in L_{p}\left(\Omega ; d^{*}(w, 1)^{(M)}\right)$.

Proof. We start by using an argument due to Muckenhoupt [15] (see also [20]). For any fixed $\varepsilon_{1}, \ldots, \varepsilon_{N}$ let $S=\sum_{k=1}^{N} \varepsilon_{k} \Delta_{k}$. Now fix $f \in L_{\infty}$. Then by a result of Burkholder [2], $\|S\|_{L_{p} \rightarrow L_{p}}=p-1$ if $2 \leq p<\infty$. Then for any $\alpha>0$ we have

$$
\mathbb{E}(\cosh (\alpha|S f|)) \leq 1+\sum_{m=1}^{\infty} \frac{\alpha^{2 m}}{(2 m) !}(2 m-1)^{2 m}\|f\|_{L_{2 m}}^{2 m} .
$$


Since $\|f\|_{L_{2 m}}^{2 m} \leq\|f\|_{L_{2}}^{2}\|f\|_{L_{\infty}}^{2 m-2}$ and since for $m \geq 1$ we have

$$
\frac{(2 m-1)^{2 m}}{(2 m) !} \leq \frac{(2 m)^{2 m}}{(2 m) !} \leq e^{2 m}
$$

it follows from (3.3) that

$$
\mathbb{E}(\cosh (\alpha|S f|)-1) \leq(\alpha e)^{2}\|f\|_{L_{2}}^{2} \sum_{k=0}^{\infty}(\alpha e)^{2 k}\|f\|_{L_{\infty}}^{2 k} .
$$

In particular, if $\alpha e\|f\|_{\infty} \leq 1 / 2$ we have

$$
\mathbb{E}(\cosh (\alpha|S f|)-1) \leq 2 e^{2} \alpha^{2}\|f\|_{L_{2}}^{2} .
$$

At this point we return to the Lorentz space $d(w, 1)$. Let us define $\gamma_{0}=0$, $\gamma_{1}=1$, and $\gamma_{k}=2^{2^{k-2}}$ for $k \geq 2$. Let $W_{k}=w_{\gamma_{k}}$. It will be convenient to normalize condition (3.2) so that we have

$$
\sum_{k=1}^{\infty} \gamma_{k} W_{k}=1
$$

We also note that (3.1) implies the existence of a constant $C$ such that

$$
\left|w_{1}+\ldots+w_{k}\right| \leq C k w_{k}
$$

for $k \geq 1$.

Now suppose $\mathbf{f}=\left(f_{j}\right)_{j=1}^{M} \in L_{\infty}\left(\Omega ; \mathbb{C}^{M}\right)$. Suppose that $\mathbf{f}$ is supported on a measurable set $E$ and satisfies $\|\mathbf{f}(\omega)\|_{d^{*}} \leq 1$ everywhere. Then we can define a measurable map $\pi$ from $\Omega$ into the set of permutations of $\{1, \ldots, M\}$ so that $\left|f_{\pi(\omega)(1)}(\omega)\right| \geq \ldots \geq\left|f_{\pi(\omega)(M)}(\omega)\right|$ for all $\omega \in \Omega$. Thus

$$
\left|f_{\pi(\omega)(j)}(\omega)\right| \leq C w_{j}
$$

for all $1 \leq j \leq M$. Let $E_{j k}=\{\omega \in E: \pi(\omega)(k)=j\}$ when $j, k \in\{1, \ldots, M\}$ and $E_{j k}=\emptyset$ otherwise. Now for $1 \leq j \leq M$ and $l=1,2, \ldots$, let

$$
f_{j}^{(l)}=\sum_{k=\gamma_{l-1}}^{\gamma_{l}-1} f_{j} \chi_{E_{j k}}
$$

so that $f_{j}=\sum_{l=1}^{\infty} f_{j}^{(l)}$. If $0<\alpha e \leq 1 /(2 C)$ we can estimate

$$
\begin{aligned}
\mathbb{E}\left(\cosh \left(\alpha\left|S f_{j}\right|\right)-1\right) & =\mathbb{E}\left(\cosh \left(\left|\sum_{l=1}^{\infty} \alpha S f_{j}^{(l)}\right|\right)-1\right) \\
& \leq \mathbb{E}\left(\max _{l \geq 1}\left(\cosh \left(\alpha \gamma_{l}^{-1} W_{l}^{-1}\left|S f_{j}^{(l)}\right|\right)-1\right)\right) \\
& \leq e^{2} \alpha^{2} \sum_{l=1}^{\infty} \gamma_{l}^{-2} W_{l}^{-2}\left\|f_{j}^{(l)}\right\|_{L_{2}}^{2}
\end{aligned}
$$


in view of (3.4) since $\left\|f_{j}^{(l)}\right\|_{L_{\infty}} \leq C W_{l}$ and $\alpha \gamma_{l}^{-1} W_{l}^{-1}\left\|f_{j}^{(l)}\right\|_{L_{\infty}} \leq 1 / 2$. Thus

$$
\mathbb{E}\left(\cosh \left(\alpha\left|S f_{j}\right|\right)-1\right) \leq e^{2} C^{2} \alpha^{2} \sum_{l=1}^{\infty} \gamma_{l}^{-2} \sum_{k=\gamma_{l-1}}^{\gamma_{l}-1} \mathbb{P}\left(E_{j k}\right) .
$$

It follows that

$$
\mathbb{E}\left(\cosh \left(\alpha\|S \mathbf{f}\|_{\ell_{\infty}}\right)-1\right) \leq e^{2} C^{2} \alpha^{2} \sum_{j=1}^{M} \sum_{l=1}^{\infty} \gamma_{l}^{-2} \sum_{k=\gamma_{l-1}}^{\gamma_{l}-1} \mathbb{P}\left(E_{j k}\right) .
$$

Note that for each $k \in \mathbb{N}, \sum_{j=1}^{M} \mathbb{P}\left(E_{j k}\right) \leq \mathbb{P}(E)$. Hence if $\mathbf{f}$ is supported on $E$ with $\|\mathbf{f}(\omega)\|_{d^{*}} \leq 1$ everywhere and $\alpha e<1 /(2 C)$, then

$$
\mathbb{E}\left(\cosh \left(\alpha\|S \mathbf{f}\|_{\ell_{\infty}}\right)-1\right) \leq e^{2} C^{2} \alpha^{2} \sum_{l=1}^{\infty} \gamma_{l}^{-1} \mathbb{P}(E)=C_{1} \alpha^{2} \mathbb{P}(E)
$$

for a suitable constant $C_{1}$. Let us next refine (3.7). For $n \geq 0$, let

$$
G_{n}=\left\{\omega \in E: 4^{-n-1}<\|\mathbf{f}(\omega)\|_{d^{*}} \leq 4^{-n}\right\} .
$$

Then by (3.7) we have, if $\alpha<(4 C e)^{-1}$,

$$
\mathbb{E}\left(\cosh \left(2^{n+1} \alpha\left\|S\left(\mathbf{f} \chi_{G_{n}}\right)\right\|_{\ell_{\infty}}\right)-1\right) \leq C_{1} \alpha^{2} 4^{-n} \mathbb{P}\left(G_{n}\right)
$$

and as

$$
\mathbb{E}\left(\cosh \left(\alpha\|S \mathbf{f}\|_{\ell_{\infty}}\right)-1\right) \leq \mathbb{E}\left(\sup _{n \geq 0}\left(\cosh \left(2^{n+1} \alpha\left\|S\left(\mathbf{f} \chi_{G_{n}}\right)\right\|_{\ell_{\infty}}\right)-1\right)\right),
$$

we obtain, under the assumptions $\|\mathbf{f}(\omega)\|_{d^{*}} \leq 1$ everywhere and $\alpha<(4 C)^{-1}$,

$$
\mathbb{E}\left(\cosh \left(\alpha\|S \mathbf{f}\|_{\ell_{\infty}}\right)-1\right) \leq C_{1} \alpha^{2} \sum_{n=0}^{\infty} 4^{-n} \mathbb{P}\left(G_{n}\right) \leq C_{2} \mathbb{E}\left(\|\mathbf{f}\|_{d^{*}}\right) .
$$

If we use a fixed value of $\alpha$ and the estimate $x^{2} \leq 2(\cosh x-1)$ we find that

$$
\mathbb{E}\left(\|S \mathbf{f}\|_{\ell_{\infty}}^{2}\right) \leq C_{3} \mathbb{E}\left(\|\mathbf{f}\|_{d^{*}}\right)
$$

if \|\| $\mathbf{f}\left\|_{d^{*}}\right\|_{\infty} \leq 1$. This in turn gives us, for every $\mathbf{f} \in L_{\infty}\left(\Omega ; d^{*}(w, 1)^{(M)}\right)$,

$$
\mathbb{E}\left(\|S \mathbf{f}\|_{\ell_{\infty}}^{2}\right) \leq C_{3}\|\| \mathbf{f}\left\|_{d^{*}}\right\|_{\infty} \mathbb{E}\left(\|\mathbf{f}\|_{d^{*}}\right) .
$$

Now let $2<p<\infty$ and fix $\mathbf{f}$ with $\mathbb{E}\left(\|\mathbf{f}\|_{d^{*}}^{p}\right)=1$. We set $E_{0}=\left\{\|\mathbf{f}\|_{d^{*}} \leq 1\right\}$ and $E_{n}=\left\{2^{n-1}<\|\mathbf{f}\|_{d^{*}} \leq 2^{n}\right\}$ for $n \geq 1$. Applying (3.9) we obtain

$$
\begin{aligned}
\left(\mathbb{E}\left(\|S \mathbf{f}\|_{\ell_{\infty}}^{2}\right)\right)^{1 / 2} & \leq\left(C_{3} \sum_{n=0}^{\infty} 2^{n} \mathbb{P}\left(E_{n}\right) \mathbb{E}\left(\|\mathbf{f}\|_{d^{*}}\right)\right)^{1 / 2} \leq C_{3}^{1 / 2} \sum_{n=0}^{\infty} 2^{n / 2} \mathbb{P}\left(E_{n}\right)^{1 / 2} \\
& \leq C_{3}^{1 / 2}\left(\sum_{n=0}^{\infty} 2^{(2-p) n}\right)^{1 / 2}\left(\sum_{n=0}^{\infty} 2^{n p} \mathbb{P}\left(E_{n}\right)\right)^{1 / 2} \leq C_{4},
\end{aligned}
$$


which completes the proof under the assumption $\mathbb{E}\left(\|\mathbf{f}\|_{d^{*}}^{p}\right)=1$. The general case follows by scaling.

We now establish our main estimate for $h(A)$.

Theorem 3.4. Let $w=\left(w_{n}\right)_{n=1}^{\infty}$ be a sequence satisfying (3.1) and (3.2). Then there is a constant $C$ so that for any $M \times N$ matrix $A=\left(a_{k j}\right)_{j, k}$ we have

$$
h(A) \leq C \max _{1 \leq k \leq N}\left\|\mathbf{a}_{k}\right\|_{d^{*}}
$$

where $\mathbf{a}_{k}=\left(a_{k j}\right)_{j=1}^{M}$. In particular, we have

$$
h(A) \leq C \max _{j, k} \frac{\left|a_{j k}\right|}{w_{|j-k|+1}} .
$$

Proof. Suppose $p>2$ and that $A$ is a matrix satisfying $\max _{1 \leq k \leq N}\left\|\mathbf{a}_{k}\right\|_{d^{*}}$ $\leq 1$. Consider the operator $T_{A}: L_{p}(\Omega) \rightarrow L_{2}\left(\Omega ; \ell_{\infty}^{M}\right)$. The adjoint operator is $T_{A}^{*}: L_{2}\left(\Omega ; \ell_{1}^{M}\right) \rightarrow L_{p^{\prime}}(\Omega)$ given by

$$
T_{A}^{*}(\mathbf{f})=\sum_{k=1}^{N}\left\langle\Delta_{k} \mathbf{f}, \mathbf{a}_{k}\right\rangle .
$$

The dual statement of the result in Proposition 3.3 entails that for any sequence $\varepsilon_{1}, \ldots, \varepsilon_{N}$ of \pm 1 's, we have the estimate

$$
\left(\mathbb{E}\left(\left\|\sum_{k=1}^{N} \varepsilon_{k} \Delta_{k} \mathbf{f}\right\|_{d}^{p^{\prime}}\right)\right)^{1 / p^{\prime}} \leq C\left(\mathbb{E}\left(\|\mathbf{f}\|_{\ell_{1}}^{2}\right)\right)^{1 / 2}
$$

where $C$ depends only on $\left(w_{n}\right)$. Now let $\varepsilon_{1}, \ldots, \varepsilon_{N}$ be a sequence of independent Bernoulli random variables on some probability space $\left(\Omega^{\prime}, \mathbb{P}^{\prime}\right)$. We use $\mathbb{E}^{\prime}$ to denote expectations on $\Omega^{\prime}$. Using Lemma 3.2 we obtain

$$
\begin{aligned}
\left(\mathbb{E}\left(\left\|T_{A}^{*} \mathbf{f}\right\|_{d}^{p^{\prime}}\right)\right)^{1 / p^{\prime}} & \leq C_{0}\left(\mathbb{E}\left(\sum_{k=1}^{N}\left|\left\langle\Delta_{k} \mathbf{f}, \mathbf{a}_{k}\right\rangle\right|^{2}\right)^{p^{\prime} / 2}\right)^{1 / p^{\prime}} \\
& \leq C_{0}\left(\mathbb{E}\left(\sum_{k=1}^{N}\left\|\Delta_{k} \mathbf{f}\right\|_{d}^{2}\right)^{p^{\prime} / 2}\right)^{1 / p^{\prime}} \\
& \leq C_{1}\left(\mathbb{E} \mathbb{E}^{\prime}\left(\left\|\sum_{k=1}^{N} \varepsilon_{k} \Delta_{k} \mathbf{f}\right\|_{d}^{p^{\prime}}\right)\right)^{1 / p^{\prime}} \leq C_{2}\left(\mathbb{E}\|\mathbf{f}\|_{\ell_{1}}^{2}\right)^{1 / 2}
\end{aligned}
$$

This gives $h_{p, 2}(A) \leq C_{2}$, which completes the proof by using Theorem 2.2.

Remark. Theorem 3.4 implies that given any $\theta>1$ there is a constant $C_{\theta}$ so that

$$
h(A) \leq C_{\theta}
$$


whenever $A=\left(a_{k j}\right)_{j, k}$ is a matrix satisfying

$$
\left|a_{j k}\right| \leq 2(\log (2+|j-k|))^{-\theta} .
$$

We show that this is not the case when $0<\theta<1 / 2$. Let $N$ be any natural number and define $A=\left(a_{j k}\right)$ to be a $2^{N} \times N$ matrix given by $a_{j k}=b_{j k} N^{-\theta}$, where $b_{j k}= \pm 1$ and the set $\left(b_{j k}\right)_{j=1}^{2^{N}}$ runs through all $2^{N}$ choices of signs. Choose $f$ real so that $\left|\Delta_{k} f\right|=1$ for $1 \leq k \leq N$. Then $\|f\|_{L_{2}}=\sqrt{N}$. On the other hand,

$$
\max _{1 \leq j \leq 2^{N}}\left|\sum_{k=1}^{N} a_{j k} \Delta_{k} f\right|=N^{1-\theta} \chi_{\Omega},
$$

which implies that $h(A) \geq N^{1 / 2-\theta}$. However,

$$
\left|a_{j k}\right| \leq N^{-\theta} \leq 2(N+1)^{-\theta} \leq 2(\log (2+|j-k|))^{-\theta}
$$

but $h(A) \geq N^{1 / 2-\theta} \rightarrow \infty$ as $N \rightarrow \infty$. Thus (3.11) fails when $0<\theta<1 / 2$.

4. The harmonic version of the maximal operator. We shall now fix $n \in \mathbb{N}$ and work with $\mathbb{R}^{n}$. Let $\mathcal{D}_{0}$ be the collection of all unit cubes of the form $\prod_{j=1}^{n}\left[m_{j}, m_{j}+1\right]$ where $m_{j} \in \mathbb{Z}$ and let $\mathcal{D}_{k}$ be the set of all cubes of the form $\prod_{j=1}^{n}\left[2^{-k} m_{j}, 2^{-k}\left(m_{j}+1\right)\right]$ where $m_{j} \in \mathbb{Z}$. For $k \in \mathbb{Z}$, let $\Sigma_{k}$ denote the $\sigma$-algebra generated by the dyadic cubes $\mathcal{D}_{k}$. We define the corresponding conditional expectation operators

$$
\mathcal{E}_{k} f=\sum_{Q \in \mathcal{D}_{k}}(\underset{Q}{\operatorname{Ave} f}) \chi_{Q}
$$

for $f \in L_{1}^{\text {loc }}\left(\mathbb{R}^{n}\right)$ and the martingale difference operators $\Delta_{k} f=\mathcal{E}_{k} f-\mathcal{E}_{k-1} f$ for $k \in \mathbb{Z}$.

Now let $A=\left(a_{j k}\right)_{j, k \in \mathbb{Z}}$ be any infinite complex matrix. We shall call $A$ a $c_{00}$-matrix if it has only finitely many non-zero entries. For a $c_{00}$-matrix $A$ define $h_{p}[A ; n]$ to be the least constant such that for all $f \in L_{p}\left(\mathbb{R}^{n}\right)$ we have

$$
\left\|\max _{j \in \mathbb{Z}}\left|\sum_{k \in \mathbb{Z}} a_{j k} \Delta_{k} f\right|\right\|_{L_{p}} \leq h_{p}[A ; n]\|f\|_{L_{p}} .
$$

Also, let $h_{p}^{\mathrm{w}}[A ; n]$ be the corresponding weak-type constant, i.e. the least constant such that for all $f \in L_{p}\left(\mathbb{R}^{n}\right)$ we have

$$
\left\|\max _{j \in \mathbb{Z}}\left|\sum_{k=1}^{N} a_{j k} \Delta_{k} f\right|\right\|_{L_{p, \infty}} \leq h_{p}^{\mathrm{w}}[A ; n]\|f\|_{L_{p}} .
$$

The following lemma is easily verified and we omit its proof.

Lemma 4.1. Let $h_{p}^{\mathrm{w}}(A ; n)$ and $h_{p}(A ; n)$ be as in Proposition 2.5. For any $1<p<\infty$ and any infinite $c_{00}$-matrix $A$ we have $h_{p}[A ; n]=h_{p}(B ; n)$ 
and $h_{p}^{\mathrm{w}}[A ; n]=h_{p}^{\mathrm{w}}(B ; n)$, where $B$ is any $M \times N$ matrix of the form $b_{j k}=$ $a_{j+r, k+s}$ for some $r, s \in \mathbb{Z}$ such that $a_{j+r, k+s}=0$ unless $1 \leq j \leq M$ and $1 \leq k \leq N$.

Now for any infinite matrix $A$ we define

$$
h(A)=\sup _{N} h\left(\left(a_{j-N, k-N}\right)_{1 \leq j, k \leq 2 N}\right) .
$$

The following is an immediate consequence of Lemma 4.1 and Proposition 2.5 .

Corollary 4.2. For any $1<p<\infty$ and any $n \in \mathbb{N}$ there is a constant $C=C(p, N)$ so that for any infinite $c_{00}$-matrix we have

$$
C^{-1} h(A) \leq h_{p}^{\mathrm{w}}[A ; n] \leq h_{p}[A ; n] \leq C h(A) .
$$

We now turn to the harmonic model of the maximal operator studied in Section 2. Let $\mathcal{S}\left(\mathbb{R}^{n}\right)$ denote the set of all Schwartz functions on $\mathbb{R}^{n}$ and for $f \in \mathcal{S}\left(\mathbb{R}^{n}\right)$ let

$$
\widehat{f}(\xi)=\int_{\mathbb{R}^{n}} f(x) e^{-2 \pi i\langle\xi, x\rangle} d x
$$

denote the Fourier transform of $f$. We will denote by $f^{\vee}(\xi)=\widehat{f}(-\xi)$ the inverse Fourier transform of $f$. We shall fix a radial function $\psi \in \mathcal{S}\left(\mathbb{R}^{n}\right)$ whose Fourier transform is real-valued and satisfies $\widehat{\psi}(\xi)=1$ for $|\xi| \leq 1$ and $\widehat{\psi}(\xi)=0$ for $|\xi| \geq 2$. We define a Schwartz function $\phi$ by setting $\widehat{\phi}(\xi)=\widehat{\psi}(\xi)-\widehat{\psi}(2 \xi)$. Then $\widehat{\phi}$ is supported in the annulus $2^{-1} \leq|\xi| \leq 2$. We then define $\psi_{j}(x)=2^{n j} \psi\left(2^{j} x\right)$ and $\phi_{j}(x)=2^{n j} \phi\left(2^{j} x\right)$ for $j \in \mathbb{Z}$. Note that $\widehat{\phi}_{j}(\xi)=\widehat{\phi}\left(2^{-j} \xi\right)$ is supported in the annulus $2^{j-1} \leq|\xi| \leq 2^{j+1}$. We also define operators

$$
\widetilde{S}_{j} f=\psi_{j} * f \quad \text { and } \quad \widetilde{\Delta}_{j} f=\phi_{j} * f
$$

for $f \in L_{1}+L_{\infty}$. The $\widetilde{\Delta}_{j}$ 's are called the Littlewood-Paley operators. Now if $A=\left(a_{j k}\right)_{(j, k) \in \mathbb{Z}^{2}}$ is an infinite $c_{00}$-matrix and $1<p<\infty$, we let $\widetilde{h}_{p}(A)$ be the least constant so that for all $f \in L_{p}$ we have

$$
\left\|\sup _{j \in \mathbb{Z}}\left|\sum_{k \in \mathbb{Z}} a_{j k} \widetilde{\Delta}_{k} f\right|\right\|_{L_{p}} \leq \widetilde{h}_{p}(A)\|f\|_{L_{p}} .
$$

We also define $\widetilde{h}_{p}^{\mathrm{w}}(A)$ to be the least constant such that for all $f \in L_{p}$ we have

$$
\left\|\sup _{j \in \mathbb{Z}}\left|\sum_{k \in \mathbb{Z}} a_{j k} \widetilde{\Delta}_{k} f\right|\right\|_{L_{p, \infty}} \leq \widetilde{h}_{p}^{\mathrm{w}}(A)\|f\|_{L_{p}} .
$$

We now have the following. 
Lemma 4.3. Suppose $r \in \mathbb{Z}$. If $1<p<\infty$ and $A=\left(a_{j k}\right)$ is any infinite $c_{00}$-matrix, then $\widetilde{h}_{p}(A)=\widetilde{h}_{p}(B)$ and $\widetilde{h}_{p}^{\mathrm{w}}(A)=\widetilde{h}_{p}^{\mathrm{w}}(B)$, where $B=\left(b_{j k}\right)$ and $b_{j k}=a_{j, k+r}$.

Proof. Consider the dilation operator $D_{r} f(x)=f\left(2^{-r} x\right)$. Then we have $D_{r}^{-1} \widetilde{\Delta}_{k} D_{r} f=\widetilde{\Delta}_{k-r} f$ and

$$
\begin{aligned}
\left\|\sup _{j}\left|\sum_{k} a_{j, k+r} \widetilde{\Delta}_{k} f\right|\right\|_{L_{p}} & =\left\|\sup _{j}\left|\sum_{k} a_{j k} \widetilde{\Delta}_{k-r} f\right|\right\|_{L_{p}} \\
& =2^{-r n / p}\left\|\sup _{j}\left|\sum_{k} a_{j k} \widetilde{\Delta}_{k} D_{r} f\right|\right\|_{L_{p}} \\
& \leq 2^{-r n / p} h_{p}(A)\left\|D_{r} f\right\|_{L_{p}}=h_{p}(A)\|f\|_{L_{p}},
\end{aligned}
$$

which implies $\widetilde{h}_{p}(B) \leq \widetilde{h}_{p}(A)$. Likewise, we obtain $\widetilde{h}_{p}(A) \leq \widetilde{h}_{p}(B)$. The corresponding result for the weak type constants follows similarly.

Next we prove that the Littlewood-Paley operators $\widetilde{\Delta}_{j}$ and the martingale difference operators $\Delta_{k}$ are essentially orthogonal on $L_{2}$ when $k \neq j$.

Proposition 4.4. There exists a constant $C$ so that for every $k, j$ in $\mathbb{Z}$ we have the following estimate on the operator norm of $\Delta_{j} \widetilde{\Delta}_{k}: L_{2}\left(\mathbb{R}^{n}\right) \rightarrow$ $L_{2}\left(\mathbb{R}^{n}\right)$ :

$$
\left\|\Delta_{k} \widetilde{\Delta}_{j}\right\|_{L_{2} \rightarrow L_{2}} \leq C 2^{-|j-k|} .
$$

Proof. By a simple dilation argument it suffices to prove (4.5) when $k=0$. In this case we have the estimate

$$
\begin{aligned}
\left\|\Delta_{0} \widetilde{\Delta}_{j}\right\|_{L_{2} \rightarrow L_{2}} & =\left\|\mathcal{E}_{0} \widetilde{\Delta}_{j}-\mathcal{E}_{-1} \widetilde{\Delta}_{j}\right\|_{L_{2} \rightarrow L_{2}} \\
& \leq\left\|\mathcal{E}_{0} \widetilde{\Delta}_{j}-\widetilde{\Delta}_{j}\right\|_{L_{2} \rightarrow L_{2}}+\left\|\mathcal{E}_{-1} \widetilde{\Delta}_{j}-\widetilde{\Delta}_{j}\right\|_{L_{2} \rightarrow L_{2}}
\end{aligned}
$$

and also by the self-adjointness of the $\Delta_{k}$ 's and $\widetilde{\Delta}_{j}$ 's we have

$$
\begin{aligned}
\left\|\Delta_{0} \widetilde{\Delta}_{j}\right\|_{L_{2} \rightarrow L_{2}} & =\left\|\widetilde{\Delta}_{j} \Delta_{0}\right\|_{L_{2} \rightarrow L_{2}}=\left\|\widetilde{\Delta}_{j} \mathcal{E}_{0}-\widetilde{\Delta}_{j} \mathcal{E}_{-1}\right\|_{L_{2} \rightarrow L_{2}} \\
& \leq\left\|\widetilde{\Delta}_{j} \mathcal{E}_{0}-\mathcal{E}_{0}\right\|_{L_{2} \rightarrow L_{2}}+\left\|\widetilde{\Delta}_{j} \mathcal{E}_{-1}-\mathcal{E}_{0}\right\|_{L_{2} \rightarrow L_{2}} .
\end{aligned}
$$

The required estimate (4.5) (when $k=0$ ) will be a consequence of the pair of inequalities

$$
\begin{array}{rr}
\left\|\mathcal{E}_{0} \widetilde{\Delta}_{j}-\widetilde{\Delta}_{j}\right\|_{L_{2} \rightarrow L_{2}}+\left\|\mathcal{E}_{-1} \widetilde{\Delta}_{j}-\widetilde{\Delta}_{j}\right\|_{L_{2} \rightarrow L_{2}} \leq C 2^{j} & \text { when } j \leq 0, \\
\left\|\widetilde{\Delta}_{j} \mathcal{E}_{0}-\mathcal{E}_{0}\right\|_{L_{2} \rightarrow L_{2}}+\left\|\widetilde{\Delta}_{j} \mathcal{E}_{-1}-\mathcal{E}_{0}\right\|_{L_{2} \rightarrow L_{2}} \leq C 2^{-j} & \text { when } j \geq 0 .
\end{array}
$$

We start by proving (4.6). We only consider the term $\mathcal{E}_{0} \widetilde{\Delta}_{j}-\widetilde{\Delta}_{j}$ since the term $\mathcal{E}_{-1} \widetilde{\Delta}_{j}-\widetilde{\Delta}_{j}$ is similar. Let $f \in L_{2}\left(\mathbb{R}^{n}\right)$. Then 


$$
\begin{aligned}
\left\|\mathcal{E}_{0} \widetilde{\Delta}_{j} f-\widetilde{\Delta}_{j} f\right\|_{L_{2}}^{2} \\
=\sum_{Q \in \mathcal{D}_{0}} \| f * \phi_{j}-\underset{Q}{\operatorname{Ave}\left(f * \phi_{j}\right) \|_{L_{2}(Q)}^{2}} \\
\leq \sum_{Q \in \mathcal{D}_{0}} \iint_{Q}\left|\left(f * \phi_{j}\right)(x)-\left(f * \phi_{j}\right)(t)\right|^{2} d t d x \\
\leq \sum_{Q \in \mathcal{D}_{0}} \iint_{Q}\left(\int_{3 Q}|f(y)| \cdot\left|\phi_{j}(x-y)\right| d y\right)^{2} d t d x \\
\quad+\sum_{Q \in \mathcal{D}_{0}} \int_{Q} \int_{Q}\left(\int_{3 Q}|f(y)| \cdot\left|\phi_{j}(t-y)\right| d y\right)^{2} d t d x \\
\quad+\sum_{Q \in \mathcal{D}_{0}} \int_{Q} \int_{Q}\left(\int_{(3 Q)^{\mathrm{c}}}|f(y)| 2^{j n+j}\left|\nabla \phi\left(2^{j}\left(\xi_{x, t}-y\right)\right)\right| d y\right)^{2} d t d x
\end{aligned}
$$

where $\xi_{x, t}$ lies on the line segment between $x$ and $t$. It is now easy to see that the sum of the last three expressions above is bounded by

$$
C 2^{2 j n} \sum_{Q \in \mathcal{D}_{0}} \int_{3 Q}|f(y)|^{2} d y+C_{M} 2^{2 j} \sum_{Q \in \mathcal{D}_{0}} \int_{Q}\left(\int_{\mathbb{R}^{n}} \frac{2^{j n}|f(y)| d y}{\left(1+2^{j}|x-y|\right)^{M}}\right)^{2} d x,
$$

which is clearly controlled by $C 2^{2 j}\|f\|_{L_{2}}^{2}$. This estimate is useful when $j \leq 0$.

We now turn to the proof of (4.7). Since $\widetilde{\Delta}_{j}$ is the difference of two $\widetilde{S}_{j}$ 's, it will suffice to prove $(4.7)$ where $\widetilde{\Delta}_{j}$ is replaced by $\widetilde{S}_{j}$. We only work with the term $\widetilde{S}_{j} \mathcal{E}_{0}-\mathcal{E}_{0}$ since the other term can be treated similarly. We have

$$
\begin{aligned}
\left\|\widetilde{S}_{j} \mathcal{E}_{0} f-\mathcal{E}_{0} f\right\|_{L_{2}}^{2}= & \left\|\sum_{Q \in \mathcal{D}_{0}}(\underset{Q}{\operatorname{Ave} f})\left(\psi_{j} * \chi_{Q}-\chi_{Q}\right)\right\|_{L_{2}}^{2} \\
\leq & \left.2 \| \sum_{Q \in \mathcal{D}_{0}} \underset{Q}{\operatorname{Ave} f}\right)\left(\psi_{j} * \chi_{Q}-\chi_{Q}\right) \chi_{3 Q} \|_{L_{2}}^{2} \\
& +2 \| \sum_{Q \in \mathcal{D}_{0}} \underset{Q}{\operatorname{Ave} f)\left(\psi_{j} * \chi_{Q}\right) \chi_{(3 Q)^{\mathrm{c}}} \|_{L_{2}}^{2}} .
\end{aligned}
$$

Since the functions appearing inside the sum in the first term above have supports with bounded overlap we obtain

$\left.\left\|\sum_{Q \in \mathcal{D}_{0}}(\underset{Q}{\operatorname{Ave}} f)\left(\psi_{j} * \chi_{Q}-\chi_{Q}\right) \chi_{3 Q}\right\|_{L_{2}}^{2} \leq C \sum_{Q \in \mathcal{D}_{0}} \underset{Q}{\operatorname{Ave}}|f|\right)^{2}\left\|\psi_{j} * \chi_{Q}-\chi_{Q}\right\|_{L_{2}}^{2}$,

and the crucial observation is that

$$
\left\|\psi_{j} * \chi_{Q}-\chi_{Q}\right\|_{L_{2}} \leq C 2^{-j},
$$


which can be easily checked using the Fourier transform. Therefore we obtain

$$
\left\|\sum_{Q \in \mathcal{D}_{0}}(\underset{Q}{\operatorname{Ave} f})\left(\psi_{j} * \chi_{Q}-\chi_{Q}\right) \chi_{3 Q}\right\|_{L_{2}}^{2} \leq C 2^{-2 j}\|f\|_{L_{2}}^{2}
$$

and the required conclusion will be proved if we can show that

$$
\left\|\sum_{Q \in \mathcal{D}_{0}}(\underset{Q}{\operatorname{Ave} f})\left(\psi_{j} * \chi_{Q}\right) \chi_{(3 Q)^{\mathrm{c}}}\right\|_{L_{2}}^{2} \leq C 2^{-2 j}\|f\|_{L_{2}}^{2} .
$$

We prove (4.8) by using a purely size estimate. Let $c_{Q}$ be the center of the dyadic cube $Q$. For $x \notin 3 Q$ we have the easy estimate

$$
\left|\left(\psi_{j} * \chi_{Q}\right)(x)\right| \leq \frac{C_{M} 2^{j n}}{\left(1+2^{j}\left|x-c_{Q}\right|\right)^{M}} \leq \frac{C_{M} 2^{j n}}{\left(1+2^{j}\right)^{M / 2}} \frac{1}{\left(1+\left|x-c_{Q}\right|\right)^{M / 2}}
$$

since both $2^{j} \geq 1,\left|x-c_{Q}\right| \geq 1$. We now control the left hand side of (4.8) by

$$
\begin{aligned}
& 2^{j(2 n-M)} \sum_{Q \in \mathcal{D}_{0}} \sum_{Q^{\prime} \in \mathcal{D}_{0}}(\underset{Q}{\operatorname{Ave}}|f|)\left(\underset{Q^{\prime}}{\operatorname{Ave}}|f|\right) \\
& \quad \times \int_{\mathbb{R}^{n}} \frac{C_{M} d x}{\left(1+\left|x-c_{Q}\right|\right)^{M / 2}\left(1+\left|x-c_{Q^{\prime}}\right|\right)^{M / 2}} \\
& \leq 2^{j(2 n-M)} \sum_{Q \in \mathcal{D}_{0}} \sum_{Q^{\prime} \in \mathcal{D}_{0}} \frac{\left(\operatorname{Ave}_{Q}|f|\right)\left(\operatorname{Ave}_{Q^{\prime}}|f|\right)}{\left(1+\left|c_{Q}-c_{Q^{\prime}}\right|\right)^{M / 4}} \\
& \quad \times \int_{\mathbb{R}^{n}} \frac{C_{M} d x}{\left(1+\left|x-c_{Q}\right|\right)^{M / 4}\left(1+\left|x-c_{Q^{\prime}}\right|\right)^{M / 4}} \\
& \leq 2^{j(2 n-M)} \sum_{Q \in \mathcal{D}_{0}} \sum_{Q^{\prime} \in \mathcal{D}_{0}} \frac{C_{M}^{\prime}}{\left(1+\left|c_{Q}-c_{Q^{\prime}}\right|\right)^{M / 4}}\left(\int_{Q}+\int_{Q^{\prime}}\right)|f(y)|^{2} d y \\
& \leq C_{M}^{\prime \prime} 2^{j(2 n-M)} \sum_{Q \in \mathcal{D}_{0}} \int_{Q}|f(y)|^{2} d y=C_{M}^{\prime \prime} 2^{j(2 n-M)}\|f\|_{L_{2}}^{2} .
\end{aligned}
$$

By taking $M$ large enough we obtain (4.8) and thus (4.7).

We have the following result relating $h(A)$ and $\widetilde{h}_{p}(A)$.

Theorem 4.5. For every $1<p<\infty$, there is a constant $C$ depending only on $\psi$ and $p$ so that for any $c_{00}$-matrix $A$ we have

$$
\frac{1}{C} h(A) \leq \widetilde{h}_{p}^{\mathrm{w}}(A) \leq \widetilde{h}_{p}(A) \leq C h(A) .
$$

Proof. Consider the operators $V_{r}, r \in \mathbb{Z}$, defined by

$$
V_{r}=\sum_{j \in \mathbb{Z}} \Delta_{j} \widetilde{\Delta}_{j+r}
$$


Then

$$
V_{r} V_{r}^{*}=\sum_{j, k} \Delta_{j} \widetilde{\Delta}_{j+r} \widetilde{\Delta}_{k+r} \Delta_{k}=\sum_{|j-k| \leq 1} \Delta_{j} \widetilde{\Delta}_{j+r} \widetilde{\Delta}_{k+r} \Delta_{k} .
$$

Hence by splitting into 3 pieces and using Proposition 4.4 we obtain the estimate

$$
\left\|V_{r}\right\|_{L_{2} \rightarrow L_{2}} \leq C 2^{-|r|} .
$$

Next pick $q$ so that $1<q<\infty$ and $1 / p=\theta / q+(1-\theta) / 2$ where $0<\theta<1$. Let $\left(\varepsilon_{j}\right)_{j \in \mathbb{Z}}$ be a sequence of independent Bernoulli random variables on some probability space $(\Omega, \mathbb{P})$. Then for $f \in L_{q}(\Omega)$ we have

$$
V_{r} f=\int_{\Omega} \sum_{j \in \mathbb{Z}} \sum_{k \in \mathbb{Z}} \varepsilon_{j}(\omega) \varepsilon_{k-r}(\omega) \Delta_{j} \widetilde{\Delta}_{k} f d \mathbb{P} .
$$

Averaging now gives $\left\|V_{r} f\right\|_{L_{q}}$

$$
\leq\left(\max _{\omega}\left\|\sum_{j \in \mathbb{Z}} \varepsilon_{j}(\omega) \Delta_{j}\right\|_{L_{q} \rightarrow L_{q}}\right)\left(\max _{\omega}\left\|\sum_{k \in \mathbb{Z}} \varepsilon_{k-r}(\omega) \widetilde{\Delta}_{k}\right\|_{L_{q} \rightarrow L_{q}}\right)\|f\|_{L_{q}} .
$$

Hence $\left\|V_{r}\right\|_{L_{q} \rightarrow L_{q}} \leq C$ where $C$ depends only on $q$ and $\psi$. Similarly $\left\|V_{r}^{*}\right\|_{L_{q} \rightarrow L_{q}} \leq C$. By interpolation we obtain $\left\|V_{r}\right\|_{L_{p} \rightarrow L_{p}},\left\|V_{r}^{*}\right\|_{L_{p} \rightarrow L_{p}} \leq$ $C 2^{-|r|(1-\theta)}$.

Finally, let us write

$$
\begin{aligned}
\sup _{j \in \mathbb{Z}}\left|\sum_{k \in \mathbb{Z}} a_{j k} \widetilde{\Delta}_{k} f\right| & =\sup _{j \in \mathbb{Z}}\left|\sum_{k \in \mathbb{Z}} a_{j k} \sum_{r \in \mathbb{Z}} \Delta_{k-r} \widetilde{\Delta}_{k} f\right| \\
& \leq \sum_{r \in \mathbb{Z}} \sup _{j \in \mathbb{Z}}\left|\sum_{k \in \mathbb{Z}} a_{j, k+r} \Delta_{k} \widetilde{\Delta}_{k+r} f\right| .
\end{aligned}
$$

Thus by Proposition 2.5,

$$
\left\|\sup _{j \in \mathbb{Z}}\left|\sum_{k \in \mathbb{Z}} a_{j k} \widetilde{\Delta}_{k} f\right|\right\|_{L_{p}} \leq C h(A) \sum_{r \in \mathbb{Z}}\left\|V_{r} f\right\|_{L_{p}} \leq C h_{p}(A)\|f\|_{L_{p}} .
$$

This shows that $\widetilde{h}_{p}(A) \leq C h(A)$.

For the converse direction we use $V_{r}^{*}$ and Lemma 4.3. We have

$$
\sup _{j \in \mathbb{Z}}\left|\sum_{k \in \mathbb{Z}} a_{j k} \Delta_{k} f\right| \leq \sum_{r \in \mathbb{Z}} \sup _{j \in \mathbb{Z}}\left|\sum_{k \in \mathbb{Z}} a_{j, k+r} \widetilde{\Delta}_{k} \Delta_{k+r} f\right|
$$

and so

$$
\left\|\sup _{j \in \mathbb{Z}}\left|\sum_{k \in \mathbb{Z}} a_{j k} \Delta_{k} f\right|\right\|_{L_{p, \infty}} \leq C \widetilde{h}_{p}^{\mathrm{w}}(A) \sum_{r \in \mathbb{Z}}\left\|V_{r}^{*} f\right\|_{L_{p}},
$$

which leads to the estimate $h(A) \leq C \widetilde{h}_{p}^{\mathrm{w}}(A)$. 
We next extend the definition of $\widetilde{h}_{p}(A)$ to the case when $0<p \leq 1$. For such $p$ 's we define $\widetilde{h}_{p}(A)$ to be the least constant so that for $f \in \mathcal{S}$ we have

$$
\left\|\sup _{j \in \mathbb{Z}}\left|\sum_{k \in \mathbb{Z}} a_{j k} \widetilde{\Delta} f\right|\right\|_{L_{p}} \leq C\|f\|_{H_{p}} .
$$

The space $H_{p}$ that appears on the right of (4.9) when $0<p \leq 1$ is the classical real Hardy space of Fefferman and Stein [7] and the expression \|\|$_{H_{p}}$ is its quasi-norm.

TheOREM 4.6. If $0<p<1$ then there is a constant $C=C(p, \psi)$ so that $C^{-1} h(A) \leq \widetilde{h}_{p}(A) \leq C h(A)$.

Proof. First we show the estimate $\widetilde{h}_{p}(A) \leq C h(A)$. Using the atomic characterization of $H_{p}$ (cf. [3]), we note that it suffices to get an estimate for a function $f \in \mathcal{S}$ supported in a cube $Q$ so that $|f(x)| \leq|Q|^{-1 / p}$ for $x \in Q$ and $\int x^{\alpha} f(x)=0$ if $|\alpha| \leq N=[n(1 / p-1)]$. It is then easy to see that for $x \notin 2 Q$,

$$
\left|\sum_{k \in \mathbb{Z}} a_{j k} \widetilde{\Delta}_{k} f(x)\right| \leq C h(A)\left|x-c_{Q}\right|^{-n-N-1},
$$

since $\left|a_{j k}\right| \leq C h(A)$ for each $j, k$. (Here $2 Q$ is the cube with twice the length and the same center $c_{Q}$, as usual.) This gives the estimate

$$
\int_{\mathbb{R}^{n} \backslash 2 Q} \sup _{j}\left|\sum_{k} a_{j k} \widetilde{\Delta}_{k} f(x)\right|^{p} d x \leq C^{p} h(A)^{p} .
$$

On the other hand,

$$
\int_{2 Q} \sup _{j}\left|\sum_{k} a_{j k} \widetilde{\Delta}_{k} f(x)\right|^{p} d x \leq C|Q|^{1-p / 2} h(A)^{p}\left(\int_{Q}|f(x)|^{2} d x\right)^{p / 2}
$$

and combining with the previous estimate we obtain $\widetilde{h}_{p}(A) \leq C h(A)$.

Complex interpolation gives $\widetilde{h}_{q}(A) \leq \widetilde{h}_{2}(A)^{\theta} \widetilde{h}_{p}(A)^{1-\theta}$ when $1<q<2$ and $1 / q=(1-\theta) / p+\theta / 2$. Since $\widetilde{h}_{q}(A) \geq C^{-1} h(A)$ we deduce the estimate $\widetilde{h}_{p}(A) \geq C^{-1} h(A)$.

5. Bilinear operators. Let $\sigma$ be a bounded measurable function on $\mathbb{R}^{n} \times \mathbb{R}^{n}$. For $f, g \in \mathcal{S}\left(\mathbb{R}^{n}\right)$ we define a bilinear operator $W_{\sigma}(f, g)$ with multiplier $\sigma$ by setting

$$
W_{\sigma}(f, g)(x)=\int_{\mathbb{R}^{n}} \int_{\mathbb{R}^{n}} \sigma(\xi, \eta) \widehat{f}(\xi) \widehat{g}(\eta) e^{2 \pi i\langle x, \xi+\eta\rangle} d \xi d \eta .
$$

If (5.1) is satisfied we say that $\sigma$ is the bilinear symbol (or multiplier) of $W_{\sigma}$. Now suppose $1<p_{1}, p_{2}<\infty$ and let $p_{0}$ be defined by $1 / p_{0}=1 / p_{1}+1 / p_{2}$. We say that $W_{\sigma}$ is strongly $\left(p_{1}, p_{2}\right)$-bounded if $W_{\sigma}$ extends to a bounded 
bilinear operator $L_{p_{1}} \times L_{p_{2}} \rightarrow L_{p_{0}}$. In this case we denote its norm by $\left\|W_{\sigma}\right\|_{L_{p_{1}} \times L_{p_{2}} \rightarrow L_{p_{0}}}$ (we define this expression to be $\infty$ if $W_{\sigma}$ is not bounded). Similarly, we say $W_{\sigma}$ is weakly $\left(p_{1}, p_{2}\right)$-bounded if it extends to a bounded bilinear operator $L_{p_{1}} \times L_{p_{2}} \rightarrow L_{p_{0}, \infty}$ and its norm is then denoted by $\left\|W_{\sigma}\right\|_{L_{p_{1}} \times L_{p_{2}} \rightarrow L_{p_{0}, \infty}}$.

We extend these definitions to the case $0<p_{1}, p_{2}<\infty$ by replacing the $L_{p}$ spaces by the corresponding Hardy spaces when $0<p_{j} \leq 1$. In the definition below we set $H_{p}=L_{p}$ for $1<p<\infty$. Given $0<p_{1}<p_{2}<\infty$ and $p_{0}$ defined by $1 / p_{0}=1 / p_{1}+1 / p_{2}$, we say that $W_{\sigma}$ is strongly $\left(p_{1}, p_{2}\right)$-bounded if it extends to a bounded bilinear operator $H_{p_{1}} \times H_{p_{2}} \rightarrow L_{p_{0}}$, and we denote its norm by $\left\|W_{\sigma}\right\|_{H_{p_{1}} \times H_{p_{2}} \rightarrow L_{p_{0}}}$. We say that $W_{\sigma}$ is weakly $\left(p_{1}, p_{2}\right)$-bounded if it extends to a bounded bilinear operator $H_{p_{1}} \times H_{p_{2}} \rightarrow L_{p_{0}, \infty}$, and in this case we denote its norm by $\left\|W_{\sigma}\right\|_{H_{p_{1}} \times H_{p_{2}} \rightarrow L_{p_{0}, \infty}}$. Now for a bounded function $\sigma$ on $\mathbb{R}^{n} \times \mathbb{R}^{n}$ and $0<p_{1}, p_{2}<\infty$ we define its corresponding strong and weak $\left(p_{1}, p_{2}\right)$-multiplier norm by $\|\sigma\|_{\mathcal{M}_{p_{1}, p_{2}}}=\left\|W_{\sigma}\right\|_{H_{p_{1}} \times H_{p_{2}} \rightarrow L_{p_{0}}} \quad$ and $\quad\|\sigma\|_{\mathcal{M}_{p_{1}, p_{2}}^{\mathrm{w}}}=\left\|W_{\sigma}\right\|_{H_{p_{1}} \times H_{p_{2}} \rightarrow L_{p_{0}, \infty}}$, where $1 / p_{0}=1 / p_{1}+1 / p_{2}$.

This definition of multiplier norm is analogous to that in the linear case. If $v \in L_{\infty}\left(\mathbb{R}^{n}\right)$, then $\|v\|_{\mathcal{M}_{p}}$ denotes the norm of $v$ as a multiplier from $H_{p}$ into $L_{p}$, that is,

$$
\|v\|_{\mathcal{M}_{p}}=\left\|M_{v}\right\|_{H_{p} \rightarrow L_{p}}, \quad \text { where } \quad M_{v} f=(v \widehat{f})^{\vee},
$$

when $0<p<\infty$. Next we mention a few properties of multipliers.

Proposition 5.1. Suppose $\sigma \in L_{\infty}\left(\mathbb{R}^{n} \times \mathbb{R}^{n}\right)$ and $0<p_{1}, p_{2}<\infty$. Then:

(i) If $\sigma^{\prime}(\xi, \eta)=\sigma\left(\xi-\xi_{0}, \eta-\eta_{0}\right)$ for some fixed $\xi_{0}, \eta_{0}$ then $\left\|\sigma^{\prime}\right\|_{\mathcal{M}_{p_{1}, p_{2}}}=$ $\|\sigma\|_{\mathcal{M}_{p_{1}, p_{2}}}$.

(ii) If $L: \mathbb{R}^{n} \rightarrow \mathbb{R}^{n}$ is an invertible linear operator and $\sigma_{L}(\xi, \eta)=$ $\sigma(L \xi, L \eta)$ then $\left\|\sigma_{L}\right\|_{\mathcal{M}_{p_{1}, p_{2}}}=\|\sigma\|_{\mathcal{M}_{p_{1}, p_{2}}}$.

(iii) If $\mu, v \in L_{\infty}\left(\mathbb{R}^{n}\right)$ and $\sigma^{\prime}(\xi, \eta)=\mu(\xi) \sigma(\xi, \eta) v(\eta)$, then

$$
\left\|\sigma^{\prime}\right\|_{\mathcal{M}_{p_{1}, p_{2}}} \leq\|\mu\|_{\mathcal{M}_{p_{1}}}\|\sigma\|_{\mathcal{M}_{p_{1}, p_{2}}}\|v\|_{\mathcal{M}_{p_{2}}} \text {. }
$$

Proof. For (i) note that

$$
W_{\sigma^{\prime}}(f, g)=e^{2 \pi i\left\langle x, \xi_{0}+\eta_{0}\right\rangle} W\left(e^{-2 \pi i\left\langle x, \xi_{0}\right\rangle} f, e^{-2 \pi i\left\langle x, \eta_{0}\right\rangle} g\right) .
$$

For (ii) note that $W_{\sigma_{L}}(f, g) \circ\left(L^{t}\right)^{-1}=W\left(f \circ\left(L^{t}\right)^{-1}, g \circ\left(L^{t}\right)^{-1}\right)$. Part (iii) is trivial.

Lemma 5.2. Let $\sigma \in L_{\infty}\left(\mathbb{R}^{n} \times \mathbb{R}^{n}\right)$. Suppose that either $p_{0} \geq 1$, or $\sigma$ is locally Riemann-integrable (i.e. continuous except on a set of measure zero). Then $\|\sigma\|_{L_{\infty}} \leq\|\sigma\|_{\mathcal{M}_{p_{1}, p_{2}}}$ whenever $p_{0}=p_{1} p_{2} /\left(p_{1}+p_{2}\right)$ and $0<p_{1}, p_{2}<\infty$. 
Proof. Suppose that $\sigma$ is locally Riemann-integrable and let $\left(\xi_{0}, \eta_{0}\right)$ be a point of continuity of $\sigma$. Then if we put $\sigma_{\lambda}^{\prime}(\xi, \eta)=\sigma\left(\xi_{0}+\lambda \xi, \eta_{0}+\lambda \eta\right)$, Proposition 5.1 gives $\left\|W_{\sigma_{\lambda}^{\prime}}\right\|_{H_{p_{1}} \times H_{p_{2}} \rightarrow L_{p_{0}}}=\left\|W_{\sigma}\right\|_{H_{p_{1}} \times H_{p_{2}} \rightarrow L_{p_{0}}}$. Now if $f, g \in \mathcal{S}$ it is easy to see that as $\lambda \rightarrow 0$ we have convergence in $L_{2}$ (and even pointwise) of $W_{\sigma_{\lambda}^{\prime}}(f, g)$ to $\sigma\left(\xi_{0}, \eta_{0}\right) f(x) g(x)$.

If $p_{0} \geq 1$ let $Q_{k}$ be a cube of side $2^{-k}$ centered at $(0,0)$ in $\mathbb{R}^{n} \times \mathbb{R}^{n}$. Let

$$
\sigma_{k}(\xi, \eta)=\frac{1}{\left|Q_{k}\right|} \int_{Q_{k}} \sigma\left(\xi+\xi_{0}, \eta+\eta_{0}\right) d \xi_{0} d \eta_{0}
$$

Proposition 5.1 and the fact that $p_{0} \geq 1$ easily imply that $\left\|W_{\sigma_{k}}\right\|_{L_{p_{1}} \times L_{p_{2}} \rightarrow L_{p_{0}}}$ $\leq\left\|W_{\sigma}\right\|_{L_{p_{1}} \times L_{p_{2}} \rightarrow L_{p_{0}}}$. Since $\sigma_{k}$ is continuous we have

$$
\left\|\sigma_{k}\right\|_{L_{\infty}} \leq\left\|W_{\sigma}\right\|_{L_{p_{1}} \times L_{p_{2}} \rightarrow L_{p_{0}}} .
$$

Taking limits as $k \rightarrow \infty$ yields the conclusion.

Next we require a lemma on series in $L_{p}$.

Lemma 5.3. Let $0<p<\infty$. Suppose that for some sequence $\left(f_{j k}\right)_{(j, k) \in \mathbb{Z}^{2}}$ of $L_{p}$ functions and for all pairs of sequences $\left(\delta_{j}\right)_{j \in \mathbb{Z}},\left(\delta_{k}^{\prime}\right)_{k \in \mathbb{Z}}$ with $\sup _{j \in \mathbb{Z}}\left|\delta_{j}\right|$ $\leq 1$ and $\sup _{j \in \mathbb{Z}}\left|\delta_{j}^{\prime}\right| \leq 1$, we have

$$
\sup _{N \in \mathbb{N}}\left\|\sum_{|j| \leq N} \sum_{|k| \leq N} \delta_{j} \delta_{k}^{\prime} f_{j k}\right\|_{L_{p}} \leq M .
$$

Then there is a constant $C=C(p)$ such that tionally),

(i) $\sup _{\left|\delta_{j}\right| \leq 1}\left\|\sum_{j \in \mathbb{Z}} \delta_{j} f_{j j}\right\|_{L_{p}} \leq C M$ (and the series converges uncondi-

(ii) $\left\|\left(\sum_{j \in \mathbb{Z}} \sum_{k \in \mathbb{Z}}\left|f_{j k}\right|^{2}\right)^{1 / 2}\right\|_{L_{p}} \leq C M$.

Proof. In fact (ii) follows immediately from Khinchin's inequality by taking two mutually independent sequences $\varepsilon_{j}, \varepsilon_{k}^{\prime}$ of Bernoulli random variables. To obtain (i), take a sequence $\varepsilon_{j}$ of Bernoulli random variables and for any finite subset $\mathcal{F} \subset \mathbb{Z}$ write

$$
\sum_{j \in \mathcal{F}} \delta_{j} f_{j j}=\sum_{j \in \mathcal{F}} \sum_{k \in \mathcal{F}} \delta_{j} \varepsilon_{j} \varepsilon_{k} f_{j k}-\sum_{\substack{j, k \in \mathcal{F} \\ j \neq k}} \delta_{j} \varepsilon_{j} \varepsilon_{k} f_{j k} .
$$

Now for all $\left|\delta_{j}\right| \leq 1$ (see also [10], proof of Theorem 4.6),

$$
\begin{aligned}
\mathbb{E}\left(\left\|\sum_{\substack{j, k \in \mathcal{F} \\
j \neq k}} \delta_{j} \varepsilon_{j} \varepsilon_{k} f_{j k}\right\|_{L_{p}}^{p}\right)^{1 / p} & \leq C\left\|\left(\sum_{\substack{j, k \in \mathcal{F} \\
j<k}}\left|\delta_{j} f_{j k}+\delta_{k} f_{k j}\right|^{2}\right)^{1 / 2}\right\|_{L_{p}} \\
& \leq C\left\|\left(\sum_{j \in \mathbb{Z}} \sum_{k \in \mathbb{Z}}\left|f_{j k}\right|^{2}\right)^{1 / 2}\right\|_{L_{p}} \leq C M
\end{aligned}
$$


by a generalization of Khinchin's inequality due to Bonami [1] and part (ii). The same estimate is also valid for $\sum_{j \in \mathcal{F}} \sum_{k \in \mathcal{F}} \delta_{j} \varepsilon_{j} \varepsilon_{k} f_{j k}$ by our assumptions. These estimates together with (5.2) give (i).

We now introduce some notation that will be useful in what follows. For $(j, k) \in \mathbb{Z}$ let $D_{j k}=\left\{(\xi, \eta): 2^{j-1} \leq|\xi| \leq 2^{j+1}, 2^{k-1} \leq|\eta| \leq 2^{k+1}\right\}$. Also, for $\theta>0$ let $D_{j k}(\theta)=\left\{(\xi, \eta): 2^{j-\theta} \leq|\xi| \leq 2^{j+\theta}, 2^{k-\theta} \leq|\eta| \leq 2^{k+\theta}\right\}$.

Proposition 5.4. For any $1<p_{1}, p_{2}<\infty$ there is a constant $C=$ $C\left(p_{1}, p_{2}\right)$ so that whenever $\left(\sigma_{j k}\right)_{j, k \in \mathbb{Z}}$ is a family of bilinear symbols with $\operatorname{supp} \sigma_{j k} \subset D_{j k}$ which satisfy

$$
\sup _{\left|\delta_{j}\right| \leq 1\left|\delta_{k}^{\prime}\right| \leq 1} \sup _{j}\left\|\sum_{j} \sum_{k} \delta_{j} \delta_{k}^{\prime} \sigma_{j k}\right\|_{\mathcal{M}_{p_{1}, p_{2}}} \leq M
$$

then the following statements are valid:

(i) For any scalar sequence $\left(\delta_{j}\right)$ with $\sup _{j}\left|\delta_{j}\right| \leq 1$ and any $r \in \mathbb{Z}$ we have

$$
\left\|\sum_{j \in \mathbb{Z}} \delta_{j} \sigma_{j, j+r}\right\|_{\mathcal{M}_{p_{1}, p_{2}}} \leq C M .
$$

(ii) For all $r \geq 3$ we have

$$
\left\|\sum_{j \in \mathbb{Z}} \sum_{k \leq j-r} \sigma_{j k}\right\|_{\mathcal{M}_{p_{1}, p_{2}}}+\left\|\sum_{k \in \mathbb{Z}} \sum_{j \leq k-r} \sigma_{j k}\right\|_{\mathcal{M}_{p_{1}, p_{2}}} \leq C\left(1+r^{\max \left(1 / p_{0}, 1\right)}\right) M .
$$

(iii) For every $r \geq 3, p_{0} \leq 1$ and for all $f, g \in \mathcal{S}$ we have

$$
\begin{aligned}
& \left\|\sum_{j \in \mathbb{Z}} \sum_{k \leq j-r} W_{\sigma_{j k}}\right\|_{L_{p_{1}} \times L_{p_{2}} \rightarrow H_{p_{0}}} \leq C\left(1+r^{\max \left(1 / p_{0}, 1\right)}\right) M, \\
& \left\|\sum_{k \in \mathbb{Z}} \sum_{j \leq k-r} W_{\sigma_{j k}}\right\|_{L_{p_{1}} \times L_{p_{2}} \rightarrow H_{p_{0}}} \leq C\left(1+r^{\max \left(1 / p_{0}, 1\right)}\right) M .
\end{aligned}
$$

Proof. For simplicity we write $W_{j k}=W_{\sigma_{j k}}$ below. (i) follows directly from Lemma 5.3. To prove (ii) and (iii) it is enough to consider the case $r=3$, since the other cases follow trivially by applying (i) and the case $r=3$. We therefore suppose $r \geq 3$ and establish both (ii) and (iii). An easy calculation gives that for $f, g$ Schwartz, $W_{j k}(f, g)$ has Fourier transform supported in the annulus $2^{j-2} \leq|\zeta| \leq 2^{j+2}$ when $k \leq j-3$. It follows that

$$
\begin{aligned}
\left\|\sum_{j \in \mathbb{Z}} \sum_{k \leq j-3} W_{j k}(f, g)\right\|_{L_{p_{0}}} & \leq\left\|\sum_{j \in \mathbb{Z}} \sum_{k \leq j-3} W_{j k}(f, g)\right\|_{H_{p_{0}}} \\
& \leq C\left\|\left(\sum_{j \in \mathbb{Z}}\left|\sum_{k \leq j-3} W_{j k}(f, g)\right|^{2}\right)^{1 / 2}\right\|_{L_{p_{0}}} \\
& \leq C \mathbb{E}\left(\left\|\sum_{j \in \mathbb{Z}} \varepsilon_{j} \sum_{k \leq j-3} W_{j k}(f, g)\right\|_{L_{p_{0}}}^{p_{0}}\right)^{1 / p_{0}}
\end{aligned}
$$


where as usual $\left(\varepsilon_{j}\right)_{j \in \mathbb{Z}}$ is a sequence of independent Bernoulli random variables. (If $p_{0}>1$ then $H_{p_{0}}=L_{p_{0}}$.) We need to control the last term in (5.3). Our hypothesis gives the estimate

$$
\mathbb{E}\left(\left\|\sum_{j \in \mathbb{Z}} \sum_{k \in \mathbb{Z}} \varepsilon_{j} W_{j k}(f, g)\right\|_{L_{p_{0}}}^{p_{0}}\right)^{1 / p_{0}} \leq C M\|f\|_{L_{p_{1}}}\|g\|_{L_{p_{2}}},
$$

while we can apply (i) to obtain

$$
\mathbb{E}\left(\left\|\sum_{j \in \mathbb{Z}} \sum_{k-j \mid \leq 2} \varepsilon_{j} W_{j k}(f, g)\right\|_{L_{p_{0}}}^{p_{0}}\right)^{1 / p_{0}} \leq C M\|f\|_{L_{p_{1}}}\|g\|_{L_{p_{2}}} .
$$

It remains to estimate

$$
\begin{aligned}
\mathbb{E}\left(\left\|\sum_{j \in \mathbb{Z}} \sum_{k \geq j+3} \varepsilon_{j} W_{j k}(f, g)\right\|_{L_{p_{0}}}^{p_{0}}\right)^{1 / p_{0}} \\
\leq \mathbb{E}\left(\left\|\sum_{j \in \mathbb{Z}} \sum_{k \geq j+3} \varepsilon_{j} W_{j k}(f, g)\right\|_{H_{p_{0}}}^{p_{0}}\right)^{1 / p_{0}} \\
\leq C \mathbb{E}\left(\left\|\sum_{k \in \mathbb{Z}} \sum_{j \leq k-3} \varepsilon_{j} W_{j k}(f, g)\right\|_{L_{p_{0}}}^{p_{0}}\right)^{1 / p_{0}} \\
\leq C \mathbb{E}\left(\left\|\sum_{k \in \mathbb{Z}} \varepsilon_{k}^{\prime} \sum_{j \leq k-3} \varepsilon_{j} W_{j k}(f, g)\right\|_{L_{p_{0}}}^{p_{0}}\right)^{1 / p_{0}}
\end{aligned}
$$

where $\varepsilon_{k}^{\prime}$ is a second (independent) sequence of independent Bernoulli random variables. Hence using again Khinchin's inequality we have

$$
\begin{aligned}
& \mathbb{E}\left(\left\|\sum_{j \in \mathbb{Z}} \sum_{k \geq j+3} \varepsilon_{j} W_{j k}(f, g)\right\|_{L_{p_{0}}}^{p_{0}}\right)^{1 / p_{0}} \\
& \quad \leq C\left\|\left(\sum_{k \in \mathbb{Z}} \sum_{j \leq k-3}\left|W_{j k}(f, g)\right|^{2}\right)^{1 / 2}\right\|_{L_{p_{0}}} \\
& \quad \leq C\left\|\left(\sum_{k \in \mathbb{Z}} \sum_{j \in \mathbb{Z}}\left|W_{j k}(f, g)\right|^{2}\right)^{1 / 2}\right\|_{L_{p_{0}}} \leq C M\|f\|_{L_{p_{1}}}\|g\|_{L_{p_{2}}}
\end{aligned}
$$

in view of Lemma 5.3. Using (5.4)-(5.6) we obtain

$$
\mathbb{E}\left(\left\|\sum_{j \in \mathbb{Z}} \varepsilon_{j} \sum_{k \leq j-3} W_{j k}(f, g)\right\|_{L_{p_{0}}}^{p_{0}}\right)^{1 / p_{0}} \leq C M\|f\|_{L_{p_{1}}}\|g\|_{L_{p_{2}}},
$$

which combined with (5.3) gives the first of the assertions (ii) and (iii) for $r=3$. The second assertions are derived similarly by symmetry.

We will need one further preliminary lemma.

Lemma 5.5. For any $1<p_{1}, p_{2}<\infty$ there is a constant $C=C\left(p_{1}, p_{2}\right)$ such that for any family of symbols $\left(\sigma_{j k}\right)_{j, k \in \mathbb{Z}}$ with $\operatorname{supp} \sigma_{j k} \subset D_{j k}$ and for 
any $C^{\infty}$ functions $\mu, v$ on the annulus $1 / 4 \leq|\xi| \leq 4$ we have

$$
\begin{aligned}
& \sup _{\left|\delta_{j}\right|\left|\delta_{k}^{\prime}\right| \leq 1} \sup _{j \in \mathbb{Z}}\left\|\sum_{k \in \mathbb{Z}} \delta_{j} \delta_{k}^{\prime} \tau_{j k}\right\|_{\mathcal{M}_{p_{1}, p_{2}}} \\
& \leq C K_{\mu} K_{v} \sup _{\left|\delta_{j}\right|\left|\delta_{k}^{\prime}\right| \leq 1} \sup _{j}\left\|\sum_{j} \sum_{k} \delta_{j} \delta_{k}^{\prime} \sigma_{j k}\right\|_{\mathcal{M}_{p_{1}, p_{2}}},
\end{aligned}
$$

where $\tau_{j k}(\xi, \eta)=\mu\left(2^{-j} \xi\right) \sigma_{j k}(\xi, \eta) v\left(2^{-k} \eta\right)$,

$$
K_{\mu}=\sup _{\substack{|\alpha| \leq m \\ 1 / 4 \leq|\xi| \leq 4}}\left|\frac{\partial^{\alpha} \mu}{\partial \xi^{\alpha}}\right|, \quad K_{v}=\sup _{\substack{|\alpha| \leq m \\ 1 / 4 \leq|\xi| \leq 4}}\left|\frac{\partial^{\alpha} v}{\partial \xi^{\alpha}}\right|,
$$

and $m=[(n+1) / 2]$.

Proof. Recalling the definition of $\phi$ from Section 4 we note that the function

$$
\left(\sum_{l=j-2}^{j+2} \widehat{\phi}_{l}(\xi)\right)\left(\sum_{l=j-2}^{k+2} \widehat{\phi}_{l}(\eta)\right)
$$

is compactly supported and is equal to 1 on the support of $\sigma_{j k}(\xi, \eta)$. For any sequence $\delta_{j}$ with $\sup \left|\delta_{j}\right| \leq 1$ we observe that

$$
\begin{gathered}
\left\|\left(\sum_{j \in \mathbb{Z}} \delta_{j} \mu\left(2^{-j} \xi\right)\right)\left(\sum_{l=j-2}^{j+2} \widehat{\phi}_{l}(\xi)\right)\right\|_{\mathcal{M}_{p_{1}}} \leq C K_{\mu}, \\
\left\|\left(\sum_{k \in \mathbb{Z}} \delta_{k}^{\prime} \mu\left(2^{-j k} \eta\right)\right)\left(\sum_{l=k-2}^{k+2} \widehat{\phi}_{l}(\eta)\right)\right\|_{\mathcal{M}_{p_{2}}} \leq C K_{v}
\end{gathered}
$$

by the Hörmander multiplier theorem. Let $U_{j_{1}, j_{2}, k_{1}, k_{2}}$ be the bilinear operator with symbol

$$
\left(\delta_{j_{1}} \mu\left(2^{-j_{1}} \xi\right) \sum_{l=j_{1}-2}^{j_{1}+2} \widehat{\phi}_{l}(\xi)\right) \sigma_{j_{2}, k_{2}}(\xi, \eta)\left(\delta_{k_{1}}^{\prime} v\left(2^{-k_{1}} \eta\right) \sum_{l=k_{1}-2}^{k_{1}+2} \widehat{\phi}_{l}(\eta)\right)
$$

for some fixed $\left|\delta_{j}\right|,\left|\delta_{k}^{\prime}\right| \leq 1$. Let

$$
M=\sup _{\left|\delta_{j}\right|\left|\delta_{k}^{\prime}\right| \leq 1}\left\|\sum_{j} \sum_{k} \delta_{j} \delta_{k}^{\prime} \sigma_{j k}\right\|_{\mathcal{M}_{p_{1}, p_{2}}}
$$

and let $\left(\varepsilon_{j}\right),\left(\varepsilon_{k}^{\prime}\right)$ be two sequences of mutually independent Bernoulli random variables. Then for $f, g \in \mathcal{S}$ we have

$$
\begin{aligned}
\mathbb{E}\left(\left\|\sum_{j_{1} \in \mathbb{Z}} \sum_{j_{2} \in \mathbb{Z}} \sum_{k_{1} \in \mathbb{Z}} \sum_{k_{2} \in \mathbb{Z}} \varepsilon_{j_{1}} \varepsilon_{j_{2}} \varepsilon_{k_{1}}^{\prime} \varepsilon_{k_{2}}^{\prime} U_{j_{1}, j_{2}, k_{1}, k_{2}}(f, g)\right\|_{L_{p_{0}}}^{p_{0}}\right)^{1 / p_{0}} \\
\leq C M K_{\mu} K_{v}\|f\|_{L_{p_{1}}}\|f\|_{L_{p_{2}}}
\end{aligned}
$$

by our hypothesis, (5.7), and (5.8). We now use Lemma 5.3 twice to deduce 
that

$$
\left\|\sum_{j \in \mathbb{Z}} \sum_{k \in \mathbb{Z}} U_{j, j, k, k}(f, g)\right\|_{L_{p_{0}}} \leq C K_{\mu} K_{v} M\|f\|_{L_{p_{1}}}\|g\|_{L_{p_{2}}} .
$$

This proves the required assertion.

6. Bilinear operators and infinite matrices. Recall from Section 4 that $\phi_{j}(x)=2^{n j} \phi\left(2^{j} x\right)$ are smooth bumps whose Fourier transforms are supported in the annuli $2^{j-1} \leq|\xi| \leq 2^{j+1}$. In this section we will consider symbols $\sigma$ of the form

$$
\sigma_{A}(\xi, \eta)=\sum_{j \in \mathbb{Z}} \sum_{k \in \mathbb{Z}} a_{j k} \widehat{\phi}_{j}(\xi) \widehat{\phi}_{k}(\eta)
$$

where $A=\left(a_{j k}\right)_{(j, k) \in \mathbb{Z}^{2}}$ is a bounded infinite matrix. We let $W_{A}=W_{\sigma_{A}}$ and $\|A\|_{\infty}=\sup _{j, k}\left|a_{j k}\right|$.

If $A$ is such an infinite matrix we define $A_{L}$ to be its lower triangle and $A_{\mathrm{U}}$ to be its upper triangle, i.e. $A_{\mathrm{L}}=\left(a_{j k} \theta_{j k}\right)_{j, k}$ and $A_{\mathrm{U}}=\left(a_{j k} \theta_{k j}\right)_{j, k}$ where $\theta_{j k}=1$ if $k<j$ and 0 otherwise. We let $A_{\mathrm{D}}$ be the diagonal $A-A_{\mathrm{U}}-A_{\mathrm{L}}$. Now define

$$
H(A)=h\left(A_{\mathrm{L}}\right)+h\left(A_{\mathrm{U}}^{\mathrm{t}}\right)+\|A\|_{\infty}
$$

Notice that $H(A) \geq\|A\|_{\infty}$ and that $H$ is a norm on the space of $\{A$ : $H(A)<\infty\}$, which makes it a Banach space.

Our objective will be to show that for any choice of $0<p_{1}, p_{2}<\infty$ we have $\left\|W_{A}\right\|_{H_{p_{1}} \times H_{p_{2}} \rightarrow L_{p_{0}}} \approx H(A)$. This will provide us with an equivalent expression for the norm of the multiplier $\sigma_{A}$ defined in (6.1).

We start by proving the simple upper estimate below.

Lemma 6.1. If $0<p_{1}, p_{2}<\infty$ there is a constant $C=C\left(p_{1}, p_{2}\right)$ so that for any matrix $A$ we have $\left\|\sigma_{A}\right\|_{\mathcal{M}_{p_{1}, p_{2}}} \leq C H(A)$.

Proof. We give the proof in the case $p_{1}, p_{2}>1$; the only real alteration for the other cases would be to replace the appropriate $L_{p_{j}}$-norm with the $H_{p_{j}}$-norm and use Theorem 4.6. Suppose $f, g \in \mathcal{S}$ and consider

$$
\begin{aligned}
W_{A}(f, g)= & \sum_{j \in \mathbb{Z}} \sum_{k \leq j-3} a_{j k} \widetilde{\Delta}_{j} f \widetilde{\Delta}_{k} g \\
& +\sum_{k \in \mathbb{Z}} \sum_{j \leq k-3} a_{j k} \widetilde{\Delta}_{j} f \widetilde{\Delta}_{k} g+\sum_{j \in \mathbb{Z}} \sum_{k=j-2}^{j+2} a_{j k} \widetilde{\Delta}_{j} f \widetilde{\Delta}_{k} g .
\end{aligned}
$$

We estimate the first term by noticing that for fixed $j$ the Fourier transform of $\widetilde{\Delta}_{j} f \sum_{k \leq j-3} a_{j k} \widetilde{\Delta}_{k} g$ is contained in the set $\left\{\zeta: 2^{j-2} \leq|\zeta| \leq 2^{j+2}\right\}$. 
Hence if $p_{0}>1$ we have

$$
\left\|\sum_{j \in \mathbb{Z}} \widetilde{\Delta}_{j} f \sum_{k \leq j-3} a_{j k} \widetilde{\Delta}_{k} g\right\|_{L_{p_{0}}} \leq C\left\|\left(\sum_{j \in \mathbb{Z}}\left|\widetilde{\Delta}_{j} f\right|^{2}\right)^{1 / 2}\left(\left|\sum_{k \leq j-3} a_{j k} \widetilde{\Delta}_{k}\right|^{2}\right)^{1 / 2}\right\|_{L_{p_{0}}} \text {. }
$$

If $0<p_{0} \leq 1$ we obtain the same estimate by noticing that

$$
\left\|\sum_{j \in \mathbb{Z}} \widetilde{\Delta}_{j} f \sum_{k \leq j-3} a_{j k} \widetilde{\Delta}_{k} g\right\|_{L_{p_{0}}} \leq\left\|\sum_{j \in \mathbb{Z}} \widetilde{\Delta}_{j} f \sum_{k \leq j-3} a_{j k} \widetilde{\Delta}_{k} g\right\|_{H_{p_{0}}}
$$

and using the corresponding square-function estimates in $H_{p_{0}}$. Now we have

$$
\begin{gathered}
\left\|\left(\sum_{j \in \mathbb{Z}}\left|\widetilde{\Delta}_{j} f\right|^{2}\left|\sum_{k \leq j-3} a_{j k} \widetilde{\Delta}_{k} g\right|^{2}\right)^{1 / 2}\right\|_{L_{p_{0}}} \\
\leq\left\|\left(\sum_{j \in \mathbb{Z}}\left|\widetilde{\Delta}_{j} f\right|^{2}\right)^{1 / 2} \sup _{j \in \mathbb{Z}}\left|\sum_{k \leq j-3} a_{j k} \widetilde{\Delta}_{k} g\right|\right\|_{L_{p_{0}}} .
\end{gathered}
$$

If we let $A_{\mathrm{LL}}$ be the matrix with entries $a_{j k}$ if $k \leq j-3$ and 0 otherwise, then $h\left(A_{\mathrm{LL}}\right) \leq h\left(A_{\mathrm{L}}\right)+h(B)$ where $B$ is the matrix with entries $a_{j k}$ if $j-2 \leq k \leq j-1$ and 0 otherwise. It is trivial to see that one has the estimate $h(B) \leq 2\|A\|_{\infty}$ so that $h\left(A_{\mathrm{LL}}\right) \leq C h\left(A_{\mathrm{L}}\right)$. Hence $(6.4)$ and Theorem 4.5 give

$$
\begin{aligned}
\left\|\sum_{j \in \mathbb{Z}} \sum_{k \leq j-3} a_{j k} \widetilde{\Delta}_{j} f \widetilde{\Delta}_{k} g\right\|_{L_{p_{0}}} & \leq C\left\|\left(\sum_{j \in \mathbb{Z}} \widetilde{\Delta}_{j} f\right)^{1 / 2}\right\|_{L_{p_{1}}}\left\|\sup _{j \in \mathbb{Z}}\left|\sum_{k \in \mathbb{Z}} a_{j k} \widetilde{\Delta}_{k} g\right|\right\|_{L_{p_{2}}} \\
& \leq C h\left(A_{\mathrm{L}}\right)\|f\|_{L_{p_{1}}}\|g\|_{L_{p_{2}}} .
\end{aligned}
$$

The same argument shows that the third term in (6.3) is controlled by $C h\left(A_{\mathrm{U}}^{\mathrm{t}}\right)\|f\|_{L_{p_{1}}}\|g\|_{L_{p_{2}}}$. The middle term in (6.3) is easy. For $-2 \leq r \leq 2$ we have

$$
\begin{aligned}
& \left\|\sum_{j \in \mathbb{Z}} a_{j, j+r} \widetilde{\Delta}_{j} f \widetilde{\Delta}_{j+r} g\right\|_{L_{p_{0}}} \\
& \quad \leq\left\|\left(\sum_{j \in \mathbb{Z}}\left|a_{j, j+r}\right| \cdot\left|\widetilde{\Delta}_{j} f\right|^{2}\right)^{1 / 2}\right\|_{L_{p_{1}}}\left\|\left(\sum_{k \in \mathbb{Z}}\left|a_{j, j+r}\right| \cdot\left|\widetilde{\Delta}_{j+r} g\right|^{2}\right)^{1 / 2}\right\| \|_{L_{p_{2}}} \\
& \quad \leq C \max _{j}\left|a_{j, j+r}\right| \cdot\|f\|_{L_{p_{1}}}\|g\|_{L_{p_{2}}} .
\end{aligned}
$$

Combining we obtain the required upper estimate: $\left\|\sigma_{A}\right\|_{\mathcal{M}_{p_{1}, p_{2}}} \leq C H(A)$.

To obtain the converse is somewhat more complicated. First we prove a general result which we will use in other situations as well.

Proposition 6.2. For any $1<p_{1}, p_{2}<\infty$ with $p_{0}=\left(1 / p_{1}+1 / p_{2}\right)^{-1}$ $\geq 1$, there is a constant $C=C\left(p_{1}, p_{2}\right)$ with the following property: Whenever $\left(\sigma_{j k}\right)_{(j, k) \in \mathbb{Z}^{2}}$ is a family of symbols with supp $\sigma_{j k} \subset D_{j k}$ which satisfy 


$$
\sup _{\left|\delta_{j}\right| \leq 1} \sup _{\left|\delta_{k}^{\prime}\right| \leq 1}\left\|\sum_{j} \sum_{k} \delta_{j} \delta_{k}^{\prime} W_{\sigma_{j k}}\right\|_{L_{p_{1}} \times L_{p_{2}} \rightarrow L_{p_{0}}} \leq M
$$

then

$$
\left\|\sigma_{A}\right\|_{\mathcal{M}_{p_{1}, p_{2}}} \leq C M
$$

where $A=\left(a_{j k}\right)_{j, k}$ and

$$
a_{j k}=\int_{\mathbb{R}^{n}} \int_{\mathbb{R}^{n}} \sigma_{j k}\left(2^{j} \xi, 2^{k} \eta\right) d \xi d \eta
$$

Proof. As before, we write $W_{j k}=W_{\sigma_{j k}}$. Consider first the case when $\sigma_{j k}=0$ unless $k \leq j-5$. Let $v$ be a $C^{\infty}$ function on $\mathbb{R}^{n}$ supported on $2^{-4} \leq|\xi| \leq 2^{4}$ and such that $v(\xi)=1$ on $2^{-3} \leq|\xi| \leq 2^{3}$. Fix $\xi_{0} \in \mathbb{R}^{n}$ and consider the symbol

$$
\tau_{j k}\left(\xi_{0} ; \xi, \eta\right)=v\left(2^{-j} \xi\right) \sigma_{j k}\left(\xi+2^{j} \xi_{0}, \eta\right) .
$$

Note that $\tau_{j k}$ is supported in $D_{j k}(4)$. Let $T_{j k}$ be the bilinear operator with symbol $\tau_{j k}$. For any sequences $\left(\delta_{j}\right)_{j \in \mathbb{Z}},\left(\delta_{k}^{\prime}\right)_{k \in \mathbb{Z}}$ with $\sup \left|\delta_{j}\right|, \sup \left|\delta_{k}^{\prime}\right| \leq 1$ and $f, g \in \mathcal{S}$ we have

$$
\left\|\sum_{j \in \mathbb{Z}} \sum_{k \in \mathbb{Z}} \delta_{j} \delta_{k}^{\prime} T_{j k}(f, g)\right\|_{L_{p_{0}}} \leq C\left\|\left(\sum_{j \in \mathbb{Z}}\left|\sum_{k \in \mathbb{Z}} \delta_{k}^{\prime} T_{j k}(f, g)\right|^{2}\right)^{1 / 2}\right\|_{L_{p_{0}}}
$$

by considering the supports of the Fourier transforms. But then, for fixed $j$,

$$
\sum_{k \in \mathbb{Z}} \delta_{k}^{\prime} T_{j k}(f, g)(x)=e^{-2 \pi i\left\langle x, 2^{j} \xi_{0}\right\rangle} \sum_{k \in \mathbb{Z}} \delta_{k} W_{j k}(f, g)(x),
$$

hence

$$
\begin{aligned}
\left\|\sum_{j \in \mathbb{Z}} \sum_{k \in \mathbb{Z}} \delta_{j} \delta_{k}^{\prime} T_{j k}(f, g)\right\|_{L_{p_{0}}} & \leq C\left\|\left(\sum_{j \in \mathbb{Z}}\left|\sum_{k \in \mathbb{Z}} \delta_{k}^{\prime} W_{j k}(f, g)\right|^{2}\right)^{1 / 2}\right\|_{L_{p_{0}}} \\
& \leq C\left\|\sum_{j \in \mathbb{Z}} \sum_{k \in \mathbb{Z}} \delta_{k}^{\prime} W_{j k}(f, g)\right\|_{H_{p_{0}}} \\
& \leq C M\|f\|_{L_{p_{1}}}\|g\|_{L_{p_{2}}}
\end{aligned}
$$

by Proposition 5.4 .

Now note that if $\left|\xi_{0}\right|>18$ then all $T_{j k}$ vanish. Since $p_{0} \geq 1$, we integrate over $\left|\xi_{0}\right| \leq 18$ to obtain symbols

$$
\tau_{j k}^{\prime}(\xi, \eta)=\int_{\left|\xi_{0}\right| \leq 18} \tau_{j k}(\xi, \eta) d \xi_{0}=v\left(2^{-j} \xi\right) \int_{\mathbb{R}^{n}} \sigma_{j k}\left(\xi+2^{j} \xi_{0}, \eta\right) d \xi_{0}
$$

with corresponding bilinear operators $T_{j k}^{\prime}$ satisfying

$$
\left\|\sum_{j \in \mathbb{Z}} \sum_{k \in \mathbb{Z}} \delta_{j} \delta_{k}^{\prime} T_{j k}^{\prime}\right\|_{L_{p_{1}} \times L_{p_{2}} \rightarrow L_{p_{0}}} \leq C M
$$

whenever $\left|\delta_{j}\right|,\left|\delta_{k}^{\prime}\right| \leq 1$. 
Note that $\tau_{j k}^{\prime}$ is supported on $D_{j k}(3)$. Also, if $2^{j-3} \leq|\xi| \leq 2^{j+3}$ we see that $\tau_{j k}^{\prime}(\xi, \eta)$ is constant in $\xi$.

Next let $O_{n}$ be the orthogonal group of $\mathbb{R}^{n}$ and let $d L$ denote the Haar measure on this group. Define

$$
\tau_{j k}^{\#}(\xi, \eta)=\int_{1 / 4}^{4} \lambda^{n-1} \int_{O_{n}} \tau_{j k}^{\prime}(\lambda L \xi, \lambda L \eta) d L d \lambda,
$$

and let $T_{j k}^{\#}$ be the corresponding bilinear operator. If $(\xi, \eta) \in D_{j k}$ we can compute that

$$
\tau_{j k}^{\#}(\xi, \eta)=c 2^{n k}|\eta|^{-n} a_{j k}
$$

where $c$ is a constant depending only on dimension. On the other hand, since $p_{0} \geq 1$, Proposition 5.1(ii) gives

$$
\left\|\sum_{j \in \mathbb{Z}} \sum_{k \in \mathbb{Z}} \delta_{j} \delta_{k}^{\prime} T_{j k}^{\#}\right\|_{L_{p_{1}} \times L_{p_{2}} \rightarrow L_{p_{0}}} \leq C M
$$

whenever $\left|\delta_{j}\right|,\left|\delta_{k}^{\prime}\right| \leq 1$.

Note that $\operatorname{supp} \tau_{j k}^{\#} \subset D_{j k}(6)$. Take $\mathbb{M}_{1}$ and $\mathbb{M}_{2}$ to be residue classes modulo 10 . Then if we replace $\delta_{j}$ by $\delta_{j} \chi_{\mathbb{M}_{1}}(j)$ and $\delta_{k}^{\prime}$ by $\delta_{k}^{\prime} \chi_{\mathbb{M}_{2}}(k)$ we obtain a bilinear operator whose symbol coincides with $a_{j k} 2^{n k}|\eta|^{-n} \delta_{j} \delta_{k}^{\prime}$ on $D_{j k}$ for $(j, k) \in \mathbb{M}_{1} \times \mathbb{M}_{2}$. Using Proposition 5.1(iii) and the multipliers $\sum_{j \in \mathbb{M}_{1}} \widehat{\phi}_{j}$ and $\sum_{k \in \mathbb{M}_{2}} \widehat{\phi}_{k}$ we find that the bilinear operator $V$ with symbol

$$
\sum_{j \in \mathbb{M}_{1}} \sum_{k \in \mathbb{M}_{2}} \delta_{j} \delta_{k}^{\prime} 2^{n k}|\eta|^{-n} a_{j k} \widehat{\phi}_{j}(\xi) \widehat{\phi}_{k}(\eta),
$$

satisfies $\|V\|_{L_{p_{1}} \times L_{p_{2}} \rightarrow L_{p_{0}}} \leq C M$. Summing over 100 different pairs of residue classes gives a similar estimate for the symbol

$$
\sum_{j \in \mathbb{Z}} \sum_{k \in \mathbb{Z}} \delta_{j} \delta_{k}^{\prime} 2^{n k}|\eta|^{-n} a_{j k} \widehat{\phi}_{j}(\xi) \widehat{\phi}_{k}(\eta)
$$

The last step is to remove the factor $2^{n k}|\eta|^{-n}$. But this can be done by using Lemma 5.5 since $|\eta|^{-n}$ is $C^{\infty}$ on $1 / 4 \leq|\eta| \leq 4$.

We will use this result to make an important estimate on the effect of translation in the computation of $\left\|W_{A}\right\|_{L_{p_{1}} \times L_{p_{2}} \rightarrow L_{p_{0}}}$. Let us define $A^{[r, s]}$ to be the matrix $\left(a_{j+r, k+s}\right)_{j, k}$.

Lemma 6.3. (i) There is a constant $C$ so that for all matrices $A$ we have

$$
\left\|\sigma_{A^{[r, s]}}\right\|_{\mathcal{M}_{2,2}} \leq C^{|r-s|}\left\|\sigma_{A}\right\|_{\mathcal{M}_{2,2}} .
$$

(ii) For all $1<p_{1}, p_{2}<\infty$ with $p_{0}=p_{1} p_{2} /\left(p_{1}+p_{2}\right) \geq 1$, there is a constant $C=C\left(p_{1}, p_{2}\right)$ so that if $\left|\delta_{j}\right|,\left|\delta_{k}^{\prime}\right| \leq 1$ then 


$$
\left\|\sum_{j \in \mathbb{Z}} \sum_{k \in \mathbb{Z}} \delta_{j} \delta_{k}^{\prime} a_{j k} \widehat{\phi}\left(2^{-j} \xi\right) \widehat{\phi}\left(2^{-k} \eta\right)\right\|_{\mathcal{M}_{p_{1}, p_{2}}} \leq C\left\|\sigma_{A}\right\|_{\mathcal{M}_{p_{1}, p_{2}}}
$$

i.e. $\left\|\sigma_{D}\right\|_{M_{p_{1}, p_{2}}} \leq C\left\|\sigma_{A}\right\|_{\mathcal{M}_{p_{1}, p_{2}}}$, where $D=\left(d_{j k}\right)_{j, k}=\left(\delta_{j} \delta_{k}^{\prime} a_{j k}\right)_{j, k}$.

Proof. It is clear from Proposition 5.1 that for any $r \in \mathbb{Z}$ we have

$$
\left\|W_{A[r, r]}\right\|_{L_{2} \times L_{2} \rightarrow L_{1}}=\left\|W_{A}\right\|_{L_{2} \times L_{2} \rightarrow L_{1}} .
$$

Thus it suffices to consider the case $r=0$ and $s= \pm 1$ and establish a bound in this case. To do this we consider the symbols

$$
\sigma_{j k}(\xi, \eta)=\sigma_{A}(\xi, \eta) \mu\left(2^{-j} \xi\right) v\left(2^{-k} \eta\right) \widehat{\phi}_{j}(\xi) \widehat{\phi}_{k}(\eta),
$$

where $\mu, v$ are $C^{\infty}$-functions satisfying $|\mu(\xi)|,|v(\eta)| \leq 1$ for all $\xi$, $\eta$. Since $\left\|\sum_{j \in \mathbb{Z}} \delta_{j} \mu\left(2^{-j} \xi\right) \widehat{\phi}_{j}(\xi)\right\|_{\mathcal{M}_{2}}$ is bounded by 3 whenever $\sup _{j}\left|\delta_{j}\right| \leq 1$, and there is a similar bound for $\sum_{k \in \mathbb{Z}} \delta_{k}^{\prime} v\left(2^{-k} \eta\right) \widehat{\phi}_{k}(\eta)$, we have an immediate estimate:

$$
\left\|\sum_{j \in \mathbb{Z}} \sum_{k \in \mathbb{Z}} \delta_{j} \delta_{k}^{\prime} W_{\sigma_{j k}}\right\|_{L_{2} \times L_{2} \rightarrow L_{1}} \leq 9\left\|W_{A}\right\|_{L_{2} \times L_{2} \rightarrow L_{1}} .
$$

Now let

$$
b_{j k}=\int_{\mathbb{R}^{n}} \int_{\mathbb{R}^{n}} \sigma_{j k}\left(2^{j} \xi, 2^{k} \eta\right) d \xi d \eta .
$$

Then we can compute

$$
b_{j k}=\sum_{r=-1}^{1} \sum_{s=-1}^{1} c_{r s} a_{j+r, k+s}
$$

where

$$
c_{r s}=\int_{\mathbb{R}^{n}} \int_{\mathbb{R}^{n}} \mu(\xi) v(\eta) \widehat{\phi}_{-r}(\xi) \widehat{\phi}_{-s}(\eta) \widehat{\phi}_{0}(\xi) \widehat{\phi}_{0}(\eta) d \xi d \eta .
$$

Since the functions $\widehat{\phi}_{r}$ for $-1 \leq r \leq 1$ are linearly independent on the support of $\widehat{\phi}_{0}$ we can use the above estimate for a linear combination of a finite number of choices of $v$ and $\xi$ so that $c_{r s}=0$ except when $r=0$ and $s=1$, so that $B=c A^{[0,1]}$ for some fixed constant $c \neq 0$. By Proposition 6.2 we have $\left\|W_{B}\right\|_{L_{2} \times L_{2} \rightarrow L_{1}} \leq C\left\|W_{A}\right\|_{L_{2} \times L_{2} \rightarrow L_{1}}$. This and the similar argument for the case $s=-1$ gives the result (i).

For (ii) we observe that the above argument actually also yields a bound on $\left\|W_{D}\right\|_{L_{2} \times L_{2} \rightarrow L_{1}}$ when $D=\left(d_{j k}\right)=\left(\delta_{j} \delta_{k}^{\prime} b_{j k}\right)$ (since $\delta_{j} \delta_{k}^{\prime} \sigma_{j k}$ also satisfies the hypotheses of Proposition 6.2). By choosing a similar linear combination we can then ensure that $b_{j k}=c a_{j k}$ and obtain the desired result.

The next step is to consider a discrete model of the bilinear operator $W_{\sigma_{A}}$. We restrict ourselves to $p_{1}=p_{2}=2$ for this, although our calculations 
can be done in more generality. If $A$ is a $c_{00}$-matrix we define $V_{A}: L_{2} \times L_{2} \rightarrow$ $L_{1}$ by

$$
V_{A}(f, g)=\sum_{j \in \mathbb{Z}} \sum_{k \in \mathbb{Z}} a_{j k} \Delta_{j} f \Delta_{k} g
$$

where $\Delta_{j}$ are the martingale difference operators as defined in Section 4 . We then have

Lemma 6.4. There is a constant $C$ so that if $A$ is a (strictly) lowertriangular matrix we have $h(A) \leq C\left\|V_{A}\right\|_{L_{2} \times L_{2} \rightarrow L_{1}}$.

Proof. This is a stopping time argument. Suppose $f \in L_{2}$ with $\|f\|_{L_{2}}=1$. Note that for each $j$ the function $f_{j}=\sum_{k \in \mathbb{Z}} a_{j k} \Delta_{k} f$ is $\Sigma_{j-1}$-measurable where $\Sigma_{j-1}$ is the $\sigma$-algebra generated by the dyadic cubes in $\mathcal{D}_{j-1}$. Fix $\lambda>0$. For each $j$ let $\mathcal{Q}_{j}$ be the collection of cubes $Q \in \mathcal{D}_{j-1}$ so that $\left|f_{j}\right|>\lambda$ on $Q$ and for each $j_{1}<j$ we have $\left|f_{j_{1}}\right| \leq \lambda$ on $Q$. It is not difficult to see that

$$
\left\{x: \max _{j \in \mathbb{Z}}\left|f_{j}(x)\right|>\lambda\right\}=\bigcup_{j \in \mathbb{Z}} \bigcup_{Q \in \mathcal{Q}_{j}} Q
$$

and this is a disjoint union. Also note the left-hand side has finite measure.

For each $j$ let $u_{j}$ be a $\Sigma_{j}$-measurable function such that $\left|u_{j}\right|=1$ everywhere and $\mathcal{E}_{j-1} u_{j}=0$. Let

$$
g=\sum_{j \in \mathbb{Z}} u_{j} \sum_{Q \in \mathcal{Q}_{j}} \chi_{Q}
$$

Then

$$
\|g\|_{L_{2}}^{2}=\left|\left\{x: \max _{j \in \mathbb{Z}}\left|f_{j}(x)\right|\right\}\right|
$$

and

$$
V_{A}(f, g)=\sum_{j \in \mathbb{Z}} f_{j} \Delta_{j} g=\sum_{j \in \mathbb{Z}} f_{j} u_{j} \sum_{Q \in \mathcal{Q}_{j}} \chi_{Q} .
$$

Hence

$$
\left|V_{A}(f, g)\right| \geq \lambda \chi_{\left(\max _{j}\left|f_{j}\right|>\lambda\right)}
$$

so that we have

$$
\lambda \mid\left\{\max _{j}\left|f_{j}\right|>\lambda\right\} \leq\left\|V_{A}\right\|_{L_{2} \times L_{2} \rightarrow L_{1}} .
$$

This implies that $h_{2}^{\mathrm{w}}(A) \leq\left\|V_{A}\right\|_{L_{2} \times L_{2} \rightarrow L_{1}}$ and the result follows from Theorem 2.1 .

We are now ready for the main result:

TheOREm 6.5. Suppose $0<p_{1}, p_{2}<\infty$. Then there is a constant $C=$ $C\left(p_{1}, p_{2}\right)$ so that for any infinite matrix $A$ we have

$$
\frac{1}{C} H(A) \leq\left\|\sigma_{A}\right\|_{\mathcal{M}_{p_{1}, p_{2}}^{\mathrm{w}}} \leq\left\|\sigma_{A}\right\|_{\mathcal{M}_{p_{1}, p_{2}}} \leq C H(A) .
$$


Proof. The upper bound is proved in Lemma 6.1 so we only need to prove the lower bound. It suffices to prove the results for the case when $A$ is a $c_{00}$-matrix. We start by considering the case $p_{1}=p_{2}=2$, when $A$ is strictly lower-triangular.

In this case let us estimate the norm of the discrete model $V_{A}$. In fact,

$$
\begin{aligned}
V_{A}(f, g) & =\sum_{j \in \mathbb{Z}} \sum_{k \in \mathbb{Z}} a_{j k} \Delta_{j} f \Delta_{k} g=\sum_{r \in \mathbb{Z}} \sum_{s \in \mathbb{Z}} \sum_{j \in \mathbb{Z}} \sum_{k \in \mathbb{Z}} a_{j k} \widetilde{\Delta}_{j-r} \Delta_{j} f \widetilde{\Delta}_{k-s} \Delta_{k} g \\
& =\sum_{r \in \mathbb{Z}} \sum_{s \in \mathbb{Z}} a_{j+r, k+s} \widetilde{\Delta}_{j} \Delta_{j+r} f \widetilde{\Delta}_{k} \Delta_{k+s} g \\
& =\sum_{r \in \mathbb{Z}} \sum_{s \in \mathbb{Z}} W_{A^{[r, s]}}\left(\sum_{j \in \mathbb{Z}} \widetilde{\Delta}_{j} \Delta_{j+r} f, \sum_{k \in \mathbb{Z}} \widetilde{\Delta}_{k} \Delta_{k+s} g\right) \\
& =\sum_{r \in \mathbb{Z}} \sum_{s \in \mathbb{Z}} W_{A^{[r, s]}}\left(V_{-r}^{*} f, V_{-s}^{*} g\right),
\end{aligned}
$$

where $V_{r}$ is defined in the proof of Theorem 4.5. Using Proposition 4.4 we obtain

$$
\left\|V_{A}\right\|_{L_{2} \times L_{2} \rightarrow L_{1}} \leq C \sum_{r \in \mathbb{Z}} \sum_{s \in \mathbb{Z}} 2^{-|r|-|s|}\left\|W_{A^{[r, s]}}\right\|_{L_{2} \times L_{2} \rightarrow L_{1}} .
$$

(All these quantities are finite since $A$ has only finitely many non-zero entries, and so there is a uniform bound on $W_{A^{[r, s]} \text {.) }}$

It follows that we have an estimate (for a suitable $C_{0}$ )

$$
h(A) \leq C_{0} \sum_{r \in \mathbb{Z}} \sum_{s \in \mathbb{Z}} 2^{-|r|-|s|}\left\|W_{A^{[r, s]}}\right\|_{L_{2} \times L_{2} \rightarrow L_{1}} .
$$

Next we estimate $H\left(A^{[r, s]}\right)$. If $s \geq r$ it is clear that $A$ remains lowertriangular and the invariance properties of $h(A)$ imply that $H\left(A^{[r, s]}\right) \leq$ $H(A)$. If $s<r$ then it is easy to estimate

$$
h\left(A_{\mathrm{L}}^{[r, s]}\right) \leq h\left(A_{\mathrm{L}}\right)+(r-s)\|A\|_{\infty}
$$

and

$$
h\left(\left(A_{\mathrm{U}}^{[r, s]}\right)^{\mathrm{t}}\right) \leq(r-s)\|A\|_{\infty} .
$$

We deduce that

$$
H\left(A^{[r, s]}\right) \leq h(A)+|r-s| \cdot\|A\|_{\infty}
$$

for all $r, s$. Thus for a suitable constant $C_{0}$ we have

$$
\left\|W_{A^{[r, s]}}\right\|_{L_{2} \times L_{2} \rightarrow L_{1}} \leq C_{1}(1+|r-s|) h(A) .
$$

Now we may pick an integer $N$ large enough so that

$$
C_{1} C_{0} \sum_{|r|>N} \sum_{|s|>N}(1+|r-s|) 2^{-|r|-|s|} \leq \frac{1}{2} .
$$


Then we can combine (6.5) and (6.6) to obtain

$$
h(A) \leq C_{2} \sum_{|r| \leq N} \sum_{|s| \leq N}\left\|W_{A^{[r, s]}}\right\|_{L_{2} \times L_{2} \rightarrow L_{1}} .
$$

At this point Lemma 6.3 gives the conclusion that

$$
h(A) \leq C\left\|W_{A}\right\|_{L_{2} \times L_{2} \rightarrow L_{1}} .
$$

Now suppose $A$ is arbitrary. If we let $W_{j k}$ be the bilinear operator with symbol $a_{j k} \widehat{\phi}_{j}(\xi) \widehat{\phi}_{k}(\eta)$, Lemma 6.3(ii) implies that we can use Proposition 5.4(ii) to deduce that $\left\|W_{A_{\mathrm{L}}}\right\|_{L_{2} \times L_{2} \rightarrow L_{1}} \leq C\left\|W_{A}\right\|_{L_{2} \times L_{2} \rightarrow L_{1}}$ for some absolute constant $C$. Thus the above argument yields $h\left(A_{\mathrm{L}}\right) \leq C\left\|W_{A}\right\|_{L_{2} \times L_{2} \rightarrow L_{1}}$. Similarly $h\left(A_{\mathrm{U}}^{\mathrm{t}}\right) \leq C\left\|W_{A}\right\|_{L_{2} \times L_{2} \rightarrow L_{1}}$ and Lemma 5.2 is enough to show that $\|A\|_{\infty} \leq C\left\|W_{A}\right\|_{L_{2} \times L_{2} \rightarrow L_{1}}$. Combining these we have the estimate

$$
H(A) \leq C\left\|W_{A}\right\|_{L_{2} \times L_{2} \rightarrow L_{1}} .
$$

The proof is completed by a simple interpolation technique. We will argue first that an estimate of the type

$$
H(A) \leq C\left(p_{1}, p_{2}\right)\left\|\sigma_{A}\right\|_{\mathcal{M}_{p_{1}, p_{2}}}
$$

for some fixed $1<p_{1}, p_{2}<\infty$ implies the estimate

$$
H(A) \leq C\left(q, p_{2}\right)\left\|\sigma_{A}\right\|_{\mathcal{M}_{p_{1}, q}^{\mathrm{w}}}
$$

for every $1<q<\infty$. We only need to consider the first case and $q \neq p_{2}$ (when $q=p_{2}$ one repeats the step). Then we may find $1<r<\infty$ and $0<\theta<1$ so that

$$
\frac{1}{p_{2}}=\frac{1-\theta}{q}+\frac{\theta}{r}
$$

The Marcinkiewicz interpolation theorem yields

$$
\left\|\sigma_{A}\right\|_{\mathcal{M}_{p_{1}, p_{2}}} \leq C\left(p_{1}, p_{2}, \theta\right)\left(\left\|\sigma_{A}\right\|_{\mathcal{M}_{p_{1}, q}^{\mathrm{w}}}\right)^{1-\theta}\left(\left\|\sigma_{A}\right\|_{\mathcal{M}_{p_{2}, r}}\right)^{\theta} .
$$

Since $\left\|\sigma_{A}\right\|_{\mathcal{M}_{p_{2}, r}} \leq C\left(p_{2}, r\right) H(A)$, using (6.10) and (6.8) we obtain estimate (6.9) as required (recall that we assume $A$ is a $c_{00}$-matrix so that all these quantities are finite).

Repeated use of this argument starting from $p_{1}=p_{2}=2$ gives the theorem in the cases $1<p_{1}, p_{2}<\infty$.

Finally, in the case where either $p_{1} \leq 1$ or $p_{2} \leq 1$ (or both) one can use complex interpolation to deduce

where $q_{1}, q_{2}>1$ and

$$
\left\|\sigma_{A}\right\|_{\mathcal{M}_{q_{1}, q_{2}}^{\mathrm{w}}} \leq C\left(\left\|\sigma_{A}\right\|_{\mathcal{M}_{p_{1}, p_{2}}^{\mathrm{w}}}\right)^{1-\theta}\left(\left\|\sigma_{A}\right\|_{\mathcal{M}_{2,2}}\right)^{\theta}
$$

$$
\frac{1}{q_{1}}=\frac{1-\theta}{p_{1}}+\frac{\theta}{2}, \quad \frac{1}{q_{2}}=\frac{1-\theta}{p_{2}}+\frac{\theta}{2} .
$$

This clearly extends the lower estimate to the cases $p_{1}, p_{2} \leq 1$. 
7. Applications to bilinear multipliers. We will now consider the boundedness of the bilinear operator $W_{\sigma}$ under conditions of Marcinkiewicz type on the symbol $\sigma$. We will say that a symbol $\sigma$ is $C^{N}$ if it is $C^{N}$ on the set $\{(\xi, \eta):|\xi|,|\eta|>0\}$. We first give an example to show that conditions (1.3) for a function $\sigma$ on $\mathbb{R}^{2 n}$ do not imply boundedness for the corresponding bilinear map on $\mathbb{R}^{n} \times \mathbb{R}^{n}$.

ExAmple. There is a $C^{\infty}$ symbol $\sigma$ so that for every pair of multi-indices $(\alpha, \beta)$ there is a constant $C_{\alpha, \beta}$ so that

$$
|\xi|^{|\alpha|}|\eta|^{|\beta|}\left|\partial_{\xi}^{\alpha} \partial_{\eta}^{\beta} \sigma(\xi, \eta)\right| \leq C_{\alpha, \beta}
$$

but $W_{\sigma}$ is not of weak type $\left(p_{1}, p_{2}\right)$ for any $0<p_{1}, p_{2}<\infty$.

Indeed, if we let $A$ be a bounded infinite matrix and $\sigma(\xi, \eta)=\sigma_{A}(\xi, \eta)$, then $\sigma$ satisfies the condition (7.1). However, $W_{A}$ is of weak type $\left(p_{1}, p_{2}\right)$ if and only if $H(A)<\infty$ by Theorem 6.5. At the end of Section 3 we showed that there are examples (with $A$ lower-triangular) where $H(A)=\infty$.

In fact, more is true. It is shown that the condition $0<\theta<1 / 2$ in (3.12) is insufficient to give a bound on $h(A)$ or $H(A)$ when $A$ is lower-triangular. This means that if $0<\theta<1 / 2$ we can construct a symbol $\sigma$ which is $C^{\infty}$, with $W_{\sigma}$ not of weak type $\left(p_{1}, p_{2}\right)$ for any $0<p_{1}, p_{2}<\infty$ and such that for each pair of multi-indices $(\alpha, \beta)$ there is a constant $C_{\alpha, \beta}$ with

$$
|\xi|^{|\alpha|}|\eta|^{|\beta|}\left|\partial_{\xi}^{\alpha} \partial_{\eta}^{\beta} \sigma(\xi, \eta)\right| \leq C_{\alpha, \beta}(\log (1+|\log | \xi|/| \eta||))^{-\theta}
$$

but $W_{\sigma}$ is not of weak type $\left(p_{1}, p_{2}\right)$ for any $p_{1}, p_{2}>0$.

These examples indicate that the Marcinkiewicz-type conditions (7.1) need to be modified if they are to imply boundedness for bilinear operators on $\mathbb{R}^{n} \times \mathbb{R}^{n}$.

In order to formulate some general results, let us introduce the following notation. For $\sigma \in L_{\infty}$ we define

$$
\|\sigma\|_{H}=\sup _{1 \leq|\xi| \leq 2} \sup _{1 \leq|\eta| \leq 2} H\left(\left(\sigma\left(2^{j} \xi, 2^{k} \eta\right)\right)_{j, k}\right) .
$$

If $\sigma$ is of class $C^{N}$ we define

$$
\|\sigma\|_{H}^{(N)}=\sum_{|\alpha| \leq N}\left\||\xi|^{|\alpha|} \partial_{\xi}^{\alpha} \sigma\right\|_{H}+\sum_{|\beta| \leq N}\left\||\eta|^{|\beta|} \partial_{\eta}^{\beta} \sigma\right\|_{H} .
$$

It will also be useful to define in this case

$$
\|\sigma\|_{\mathcal{M}_{p_{1}, p_{2}}}^{(N)}=\sum_{|\alpha| \leq N}\left\||\xi|^{|\alpha|} \partial_{\xi}^{\alpha} \sigma\right\|_{\mathcal{M}_{p_{1}, p_{2}}}+\sum_{|\beta| \leq N}\left\||\eta|^{|\beta|} \partial_{\eta}^{\beta} \sigma\right\|_{\mathcal{M}_{p_{1}, p_{2}}} .
$$

Now consider an arbitrary $L^{\infty}$ symbol $\sigma$ of class $C^{n+1}$. Let

$$
\sigma_{j k}(\xi, \eta)=\sigma(\xi, \eta) \widehat{\phi}\left(2^{-j} \xi\right) \widehat{\phi}\left(2^{-k} \eta\right)
$$


Set $\widehat{\zeta}(\xi)=\widehat{\phi}_{-2}(\xi)+\widehat{\phi}_{-3}(\xi)+\widehat{\phi}_{-4}(\xi)$. Then $\widehat{\zeta}$ is equal to 1 on the annulus $1 / 16 \leq|\xi| \leq 1 / 4$ and vanishes off the annulus $1 / 32 \leq|\xi| \leq 1 / 2$. Thus the function $\widehat{\zeta}(\xi) \widehat{\zeta}(\eta)$ is supported in the unit cube $[0,1]^{2 n}$ and is equal to one on the support of

$$
(\xi, \eta) \mapsto \sigma_{j k}\left(2^{j+3} \xi, 2^{k+3} \eta\right),
$$

which is also contained in $[0,1]^{2 n}$. Inspired by [5], we expand the function above in a Fourier series on $[0,1]^{2 n}$. We have

$$
\sigma_{j k}\left(2^{j+3} \xi, 2^{k+3} \eta\right)=\sum_{\nu \in \mathbb{Z}^{n}} \sum_{\varrho \in \mathbb{Z}^{n}} a_{j k}(\nu, \varrho) e^{2 \pi i(\langle\xi, \nu\rangle+\langle\eta, \varrho\rangle)} \widehat{\zeta}(\xi) \widehat{\zeta}(\eta),
$$

where for $(\nu, \varrho) \in \mathbb{Z}^{n} \times \mathbb{Z}^{n}$ we set

$$
a_{j k}(\nu, \varrho)=\int_{\mathbb{R}^{n}} \int_{\mathbb{R}^{n}} \sigma\left(2^{j+3} t, 2^{k+3} s\right) \widehat{\phi}(8 t) \widehat{\phi}(8 s) e^{-2 \pi i(\langle t, \nu\rangle+\langle s, \varrho\rangle)} d t d s .
$$

We will denote by $A(\nu, \varrho)$ the matrix with entries $a_{j k}(\nu, \varrho)$. Now setting

$$
\begin{aligned}
\tau^{\nu, \varrho}(\xi, \eta) & =\left(\sum_{j \in \mathbb{Z}} \sum_{k \in \mathbb{Z}} a_{j k}(\nu, \varrho) e^{(\pi i / 4)\left(2^{-j}\langle\xi, \nu\rangle+2^{-k}\langle\eta, \varrho\rangle\right)}\right) \widehat{\zeta}\left(2^{-j-3} \xi\right) \widehat{\zeta}\left(2^{-k-3} \eta\right),
\end{aligned}
$$

we can write a symbol $\sigma$ of class $C^{n+1}$ as

$$
\sigma(\xi, \eta)=\sum_{\nu \in \mathbb{Z}^{n}} \sum_{\varrho \in \mathbb{Z}^{n}} \tau^{\nu, \varrho}(\xi, \eta)
$$

In the next lemma we obtain some elementary estimates based on this expansion.

Lemma 7.1. Suppose $0<p_{1}, p_{2}<\infty$ and $1 / p_{0}=1 / p_{1}+1 / p_{2}$. Then:

(i) There is a constant $C=C\left(p_{1}, p_{2}\right)$ so that for any $(\nu, \varrho)$,

$$
\left\|\tau^{\nu, \varrho}\right\|_{\mathcal{M}_{p_{1}, p_{2}}} \leq C(1+|\nu|+|\varrho|)^{2 m} H(A(\nu, \varrho))
$$

where $m=[(n+1) / 2]$.

(ii) There is a constant $C=C\left(N, p_{1}, p_{2}\right)$ such that if $\sigma$ is of class $C^{N}$, and $|\nu|+|\varrho|>0$, then

$$
H(A(\nu, \varrho)) \leq C(1+|\nu|+|\varrho|)^{-N}\|\sigma\|_{H}^{(N)},
$$

while

$$
H(A(0,0)) \leq C\|\sigma\|_{H} .
$$

(iii) If $p_{0} \geq 1$ and $\sigma$ is of class $C^{N}$ then there is a constant $C=$ $C\left(N, p_{1}, p_{2}\right)$ such that

$$
H(A(\nu, \varrho)) \leq C(1+|\nu|+\mid \varrho)^{2 m-N}\|\sigma\|_{\mathcal{M}_{p_{1}, p_{2}}}^{(N)} .
$$


Proof. Observe that $\widehat{\zeta}\left(2^{-j-3} \xi\right)=\widehat{\phi}\left(2^{-j-1} \xi\right)+\widehat{\phi}\left(2^{-j} \xi\right)+\widehat{\phi}\left(2^{-j+1} \xi\right)$ and therefore $\tau^{\nu, \varrho}(\xi, \eta)$ is the sum of nine terms of the form

$$
\sum_{j \in \mathbb{Z}} \sum_{k \in \mathbb{Z}} a_{j, k}(\nu, \varrho)\left(e^{(\pi i / 4)\left\langle 2^{-j} \xi, \nu\right\rangle} \widehat{\phi}\left(2^{-j-r} \xi\right)\right)\left(e^{(\pi i / 4)\left\langle 2^{-k} \eta, \varrho\right\rangle} \widehat{\phi}\left(2^{-k-s} \eta\right)\right)
$$

where $r, s \in\{-1,0,+1\}$. We now use Lemmas 5.5, 6.3(ii) and 6.1 in that order to obtain

$$
\left\|\tau^{\nu, \varrho}\right\|_{\mathcal{M}_{p_{1}, p_{2}}} \leq C(1+|\nu|)^{m}(1+|\varrho|)^{m} H(A(\nu, \varrho))
$$

where $m=[(n+1) / 2]$. This proves $(\mathrm{i})$.

For (ii) note that if $|\alpha|,|\beta| \leq N$ integration by parts gives

$$
\begin{aligned}
& a_{j k}(\nu, \varrho) \\
& \quad=\int_{\mathbb{R}^{n}} \int_{\mathbb{R}^{n}} \partial_{\xi}^{\alpha}\left(\sigma\left(2^{j+3} \xi, 2^{k+3} \eta\right) \widehat{\phi}(8 \xi) \widehat{\phi}(8 \eta)\right) \frac{e^{-2 \pi i(\langle\xi, \nu\rangle+\langle\eta, \varrho\rangle)}}{(-2 \pi i \nu)^{\alpha}} d \xi d \eta,
\end{aligned}
$$

(7.11) $\quad a_{j k}(\nu, \varrho)$

$$
=\int_{\mathbb{R}^{n}} \int_{\mathbb{R}^{n}} \partial_{\eta}^{\beta}\left(\sigma\left(2^{j+3} \xi, 2^{k+3} \eta\right) \widehat{\phi}(8 \xi) \widehat{\phi}(8 \eta)\right) \frac{e^{-2 \pi i(\langle\xi, \nu\rangle+\langle\eta, \varrho\rangle)}}{(-2 \pi i \varrho)^{\beta}} d \xi d \eta,
$$

provided $\nu_{1}^{\alpha_{1}} \ldots \nu_{n}^{\alpha_{n}}$ and $\varrho^{\beta_{1}} \ldots \varrho_{n}^{\beta_{n}}$ are non-zero.

Now using the fact that $H$ is a norm it is easy to see that by choosing an appropriate $\alpha$ or $\beta$ for each pair $(\nu, \varrho) \neq(0,0)$ one obtains the estimate

$$
H(A(\nu, \varrho)) \leq C\left(N, p_{1}, p_{2}\right)(1+|\nu|+|\varrho|)^{-N}\|\sigma\|_{H}^{(N)}
$$

If $(\nu, \varrho)=(0,0)$ the same estimate follows directly from $(7.7)$.

Finally we turn to (iii). For fixed $\delta_{j}, \delta_{k}^{\prime}$ with $\sup \left|\delta_{j}\right|, \sup \left|\delta_{k}^{\prime}\right| \leq 1$ define $\mu(\xi)=\sum_{j \in \mathbb{Z}} \delta_{j} \widehat{\phi}_{j}(\xi)$ and $v(\eta)=\sum_{k \in \mathbb{Z}} \delta_{k}^{\prime} \widehat{\phi}_{j}(\eta)$. Then it follows from Lemma 5.5 that for any multi-indices $\alpha, \alpha^{\prime}$ we have

$$
\left\||\xi|^{|\alpha|+\left|\alpha^{\prime}\right|} \partial_{\xi}^{\alpha} \mu(\xi) \partial_{\xi}^{\alpha^{\prime}} \sigma(\xi, \eta) v(\eta)\right\|_{\mathcal{M}_{p_{1}, p_{2}}} \leq C\left(\alpha, \alpha^{\prime}\right)\|\sigma\|_{\mathcal{M}_{p_{1}, p_{2}}}^{\left(\left|\alpha^{\prime}\right|\right)} .
$$

This implies that for fixed $N$ and any $\alpha$ with $|\alpha|=N$ we have

$$
\sup _{\left|\delta_{j}\right| \leq 1} \sup _{\left|\delta_{k}^{\prime}\right| \leq 1}\left\||\xi|^{N} \sum_{j \in \mathbb{Z}} \sum_{k \in \mathbb{Z}} \delta_{j} \delta_{k}^{\prime} \partial_{\xi}^{\alpha} \sigma_{j k}(\xi, \eta)\right\|_{\mathcal{M}_{p_{1}, p_{2}}} \leq C(N)\|\sigma\|_{\mathcal{M}_{p_{1}, p_{2}}}^{(N)} .
$$

Now we either use $(7.7)$ if $(\nu, \varrho)=(0,0)$ or refer back to Proposition 6.2 (7.10) or $(7.11)$, according to the values of $\nu$ or $\varrho$, when $(\nu, \varrho) \neq(0,0)$. For example, if $N=|\nu| \geq|\varrho|$ and the $l$ th entry of $\nu$ has maximal size $N$, then

$$
\begin{aligned}
& \left\|\sum_{j \in \mathbb{Z}} \sum_{k \in \mathbb{Z}} a_{j k}(\nu, \varrho) \widehat{\phi}_{j}(\xi) \widehat{\phi}_{k}(\eta)\right\|_{\mathcal{M}_{p_{1}, p_{2}}} \\
& \leq C \sup _{\left|\delta_{j}\right| \leq 1} \sup _{\left|\delta_{k}^{\prime}\right| \leq 1}\left\|\sum_{j \in \mathbb{Z}} \sum_{k \in \mathbb{Z}} \delta_{j} \delta_{k}^{\prime} 2^{j N} \frac{\partial^{N}}{\partial \xi_{l}^{N}} \sigma_{j k}(\xi, \eta) \frac{e^{-2 \pi i\left(\left\langle 2^{-j} \xi, \nu\right\rangle+\left\langle 2^{-k} \eta, \varrho\right\rangle\right)}}{\left(-2 \pi i \nu_{l}\right)^{N}}\right\|_{\mathcal{M}_{p_{1}, p_{2}}} .
\end{aligned}
$$


Now by Lemma 5.5 we can estimate the last expression above by

$$
C(1+|\nu|+|\varrho|)^{2 m-N} \sup _{\left|\delta_{j}\right| \leq 1\left|\delta_{k}^{\prime}\right| \leq 1}\left\|\sum_{j \in \mathbb{Z}} \sum_{k \in \mathbb{Z}} \delta_{j} \delta_{k}^{\prime}|\xi|^{N} \frac{\partial^{N}}{\partial \xi_{l}^{N}} \sigma_{j k}(\xi, \eta)\right\|_{\mathcal{M}_{p_{1}, p_{2}}} .
$$

Using (7.12) we obtain (iii).

Let us state the main result of this section.

Theorem 7.2. Suppose $0<p_{1}, p_{2}<\infty$ and $1 / p_{0}=1 / p_{1}+1 / p_{2}$. Let $N=2 n+1$ if $p_{0} \geq 1$ and $N=n+2+\left[n / p_{0}\right]$ if $p_{0}<1$. Then for any $C^{N}$ symbol $\sigma$ such that $\|\sigma\|_{H}^{(N)}<\infty$ we have $\|\sigma\|_{\mathcal{M}_{p_{1}, p_{2}}}<\infty$. Furthermore,

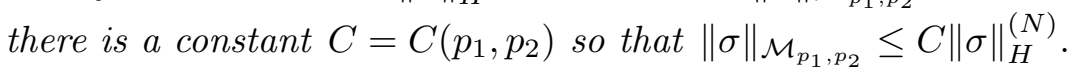

Proof. This follows directly from Lemma 7.1 and (7.9). Indeed, we have

$$
\left\|\tau^{\nu, \varrho}\right\|_{\mathcal{M}_{p_{1}, p_{2}}} \leq C(1+|\nu|+|\varrho|)^{2 m-N} \text {. }
$$

If $t=\min \left(p_{0}, 1\right)$ we have

$$
\|\sigma\|_{\mathcal{M}_{p_{1}, p_{2}}} \leq C\left(\sum_{\nu \in \mathbb{Z}} \sum_{\varrho \in \mathbb{Z}}(1+|\nu|+|\varrho|)^{(2 m-N) t}\right)^{1 / t}\|\sigma\|_{H}^{(N)} .
$$

Since $(N-2 m) t>n$ this gives the result.

We next show that in a certain sense the preceding theorem is best possible.

Theorem 7.3. Suppose $1<p_{1}, p_{2}<\infty$ and $1 / p_{0}=1 / p_{1}+1 / p_{2} \leq 1$. Suppose $\sigma$ is a $C^{\infty}$ symbol. Then the following are equivalent:

(i) $\|\sigma\|_{\mathcal{M}_{p_{1}, p_{2}}}^{(N)}<\infty$ for every $N \geq 0$.

(ii) $\|\sigma\|_{H}^{(N)}<\infty$ for every $N \geq 0$.

Proof. Assume (i); then it follows from Lemma 7.1 that for any $N>0$ we have an estimate $H(A(\nu, \varrho)) \leq C_{N}(1+|\nu|+|\varrho|)^{-N}$. Now it is clear from the definition and from Theorem 6.5 and Lemma 6.3 that we have an estimate

$$
\left\||\xi|^{|\alpha|} \partial_{\xi}^{\alpha} \tau^{\nu, \varrho}\right\|_{H} \leq C_{\alpha}(1+|\nu|)^{|\alpha|} H(A(\nu, \varrho))
$$

Hence we can easily deduce that

$$
\left\||\xi|^{\alpha} \partial_{\xi}^{\alpha} \sigma\right\|_{H}<\infty
$$

for each multi-index $\alpha$. Repeating the same reasoning with the second variable $\eta$ gives (ii).

Now assume (ii). Then for any multi-indices $\alpha, \beta$ one can easily see by differentiation that (ii) is satisfied by the symbols $|\xi|^{|\alpha|} \partial_{\xi}^{\alpha} \sigma$ and $|\eta|^{|\beta|} \partial_{\eta}^{\beta} \sigma$ in place of $\sigma$. Applying Theorem 7.2 gives (i). 
Now let us recast Theorem 7.2 in terms of estimates on the symbol $\sigma$ using the results of Section 3.

Theorem 7.4. Suppose $0<p_{1}, p_{2}<\infty$ and $1 / p_{0}=1 / p_{1}+1 / p_{2}$. Let $N=2 n+1$ if $p_{0} \geq 1$ and $N=n+2+\left[n / p_{0}\right]$ if $p_{0}<1$. Suppose that $\theta>1$ and $\sigma$ is a $C^{N}$ symbol such that for any multi-indices $\alpha, \beta$ with $0 \leq|\alpha| \leq N$ and $0 \leq|\beta| \leq N$ there exist constants $C_{\alpha}, C_{\beta}$ with

$$
\begin{aligned}
& |\xi|^{|\alpha|}\left|\partial_{\xi}^{\alpha} \sigma(\xi, \eta)\right| \leq C_{\alpha}(\log (1+|\log | \xi|/| \eta||))^{-\theta}, \\
& |\eta|^{|\beta|}\left|\partial_{\eta}^{\beta} \sigma(\xi, \eta)\right| \leq C_{\beta}(\log (1+|\log | \xi|/| \eta||))^{-\theta} .
\end{aligned}
$$

Then $\|\sigma\|_{\mathcal{M}_{p_{1}, p_{2}}}<\infty$.

REMARK. We have already seen in (7.2) that this is false when $0<\theta<$ $1 / 2$. However the arguments of Section 3 show that we can improve (7.13) and (7.14) somewhat. For example, we can replace $(\log (1+|\log | \xi|/| \eta||))^{-\theta}$ where $\theta>1$ by $(\log (1+|\log | \xi|/| \eta||))^{-1}(\log (1+\log (1+|\log | \xi|/| \eta||)))^{-\gamma}$ where $\gamma>1$.

Proof of Theorem 7.4. This follows immediately from Theorems 7.2 and 3.4 which yield the estimate

$$
H(A) \leq C \sup _{j, k}\left|a_{j k}\right| / w_{|j-k|+1}
$$

with $w_{k}=(\log (1+k))^{-\theta}$.

It is possible to "mix and match" the estimates in Section 3: for example, in the following theorem we remove the conditions for $|\alpha|,|\beta|=0$ but insist on a stronger condition for $|\alpha|=|\beta|=1$ :

Theorem 7.5. Suppose $0<p_{1}, p_{2}<\infty$ and $1 / p_{0}=1 / p_{1}+1 / p_{2}$. Let $N=2 n+1$ if $p_{0} \geq 1$ and $N=n+2+\left[n / p_{0}\right]$ if $p_{0}<1$. Suppose that $\theta>1$ and $\sigma$ is a $C^{N}$ symbol which satisfies conditions (7.13) and (7.14) for $2 \leq|\alpha|,|\beta| \leq N$, and if $|\alpha|=|\beta|=1$,

$$
\begin{aligned}
& |\xi|^{|\alpha|}\left|\partial_{\xi}^{\alpha} \sigma(\xi, \eta)\right| \leq C_{\alpha}(1+|\log | \xi|/| \eta||)^{-\theta} \\
& |\eta|^{|\beta|}\left|\partial_{\eta}^{\beta} \sigma(\xi, \eta)\right| \leq C_{\beta}(1+|\log | \xi|/| \eta||)^{-\theta}
\end{aligned}
$$

Then $\|\sigma\|_{\mathcal{M}_{p_{1}, p_{2}}}<\infty$.

Proof. It is only necessary to show that $\|\sigma\|_{H}<\infty$. Note first that Proposition 3.1 can be used to give the following estimate for any infinite matrix:

$$
H(A) \leq C\left(\|A\|_{\infty}+\sup _{j} \sum_{k<j}\left|a_{j, k}-a_{j, k+1}\right|+\sup _{k} \sum_{j<k}\left|a_{j, k}-a_{j+1, k}\right|\right) .
$$

Now suppose $1 \leq|\xi|,|\eta| \leq 2$. Then if $k<j$,

$$
\left|\sigma\left(2^{j} \xi, 2^{k} \eta\right)-\sigma\left(2^{j} \xi, 2^{k+1} \eta\right)\right| \leq C k^{-\theta}
$$

by (7.16). Combining with a similar estimate from (7.15) gives the theorem. 
We conclude this section with a theorem of the type of Theorem 7.2 for operators on $L_{1}$.

Theorem 7.6. Suppose $N=2 n+3$ and that $\sigma$ is a $C^{N}$ symbol with $\|\sigma\|_{H}^{(N)}<\infty$. Then $W_{\sigma}: L_{1} \times L_{1} \rightarrow L_{1 / 2, \infty}$ is bounded.

Proof. Let $Q$ be the cube $\left\{x: \max _{k}\left|x_{k}\right| \leq 1\right\}$ and consider the bilinear operator $W_{\sigma, Q}(f, g)=\chi_{Q} W_{\sigma}(f, g)$. We will show that if $r<1 / 2$ is such that $n+2+[n /(2 r)]=N$, then $W_{\sigma, Q}: L_{1}(2 Q) \times L_{1}(2 Q) \rightarrow L_{r}(Q)$ is bounded and $\left\|W_{\sigma, Q}\right\| \leq C\|\sigma\|_{H}^{(N)}$ where $C$ is a constant depending only on dimension.

Suppose that $f, g \in \mathcal{S}$ are functions with support contained in $2 Q$ and such that $\int f(x) d x=\int g(x) d x=0$. Then $f, g \in H_{2 r}$ with $\|f\|_{H_{2 r}} \leq C\|f\|_{L_{1}}$ and $\|g\|_{H_{2 r}} \leq C\|g\|_{L_{1}}$. Applying Theorem 7.2 we obtain

$$
\left\|W_{\sigma}(f, g)\right\|_{L_{r}} \leq C\|\sigma\|_{H}^{(N)}\|f\|_{L_{1}}\|g\|_{L_{1}}
$$

where $C$ is an absolute constant. It follows that $W_{\sigma}$ extends unambiguously to any $f, g \in L_{1}(2 Q)$ with $\int f(x) d x=\int g(x) d x=0$ and (7.17) holds.

Next fix $\psi \in \mathcal{S}$ so that $\int \psi(x) d x=1$ and $\psi$ has support contained in $Q$. Now for any $f, g \in L_{1}(3 Q)$ let $f_{0}=f-\left(\int f(x) d x\right) \psi$ and $g_{0}=g-\left(\int g(x) d x\right) \psi$. Then (7.17) gives

$$
\left\|W_{\sigma, Q}\left(f_{0}, g_{0}\right)\right\|_{L_{r}} \leq C\|\sigma\|_{H}^{(N)}\|f\|_{L_{1}}\|g\|_{L_{1}} .
$$

We also note that $\left\|W_{\sigma, Q}(\psi, \psi)\right\|_{L_{r}} \leq C\|\sigma\|_{H}^{(N)}$. Now consider the linear map $T f=W_{\sigma}(f, \psi)$. Since $\psi \in L_{2}$ we see that, if $1 / s=1 /(2 r)+1 / 2$, then $T: H_{2 r} \rightarrow L_{s}$ is bounded with norm controlled by $C\|\sigma\|_{H}^{(N)}$ (again using Theorem 7.2). Hence since $r<s$,

Similarly,

$$
\left\|W_{\sigma, Q}\left(f_{0}, \psi\right)\right\|_{L_{r}} \leq C\|\sigma\|_{H}^{(N)}\|f\|_{L_{1}} .
$$

$$
\left\|W_{\sigma, Q}\left(\psi, g_{0}\right)\right\|_{L_{r}} \leq C\|\sigma\|_{H}^{(N)}\|g\|_{L_{1}} .
$$

Combining these estimates gives

$$
\left\|W_{\sigma, Q}(f, g)\right\|_{L_{r}} \leq C\|\sigma\|_{H}^{(N)}\|f\|_{L_{1}}\|g\|_{L_{1}} .
$$

We now use a Nikishin type argument as earlier in Lemma 2.3. Suppose $\left(f_{j}\right)_{j=1}^{J}$ and $\left(g_{j}\right)_{j=1}^{J}$ satisfy $\left\|f_{j}\right\|_{L_{1}},\left\|g_{j}\right\|_{L_{1}} \leq 1$ and that $\sum_{j=1}^{J}\left|b_{j}\right|^{1 / 2}=1$. Then if $\left(\varepsilon_{j}\right)_{j=1}^{J}$ and $\left(\varepsilon_{j}^{\prime}\right)_{j=1}^{J}$ are two independent sequences of Bernoulli random variables we have

$$
\left(\mathbb{E}\left(\left\|\sum_{j=1}^{J} \sum_{k=1}^{J} \varepsilon_{j} \varepsilon_{k}^{\prime}\left|b_{j}\right|^{1 / 2}\left|b_{k}\right|^{1 / 2} W_{\sigma, Q}\left(f_{j}, g_{k}\right)\right\|_{L_{r}}^{r}\right)\right)^{1 / r} \leq C\|\sigma\|_{H}^{(N)} .
$$

Again by using the result of Bonami [1], we obtain an estimate

$$
\left\|\left(\sum_{j=1}^{J} \sum_{k=1}^{J}\left|b_{j}\right| \cdot\left|b_{k}\right| \cdot\left|W_{\sigma, Q}\left(f_{j}, g_{k}\right)\right|^{2}\right)^{1 / 2}\right\|_{L_{r}} \leq C\|\sigma\|_{H}^{(N)} .
$$


Extracting the diagonal gives

$$
\left\|\max _{1 \leq j \leq J}\left|b_{j}\right| \cdot\left|W_{\sigma, Q}\left(f_{j}, g_{j}\right)\right|\right\|_{L_{r}} \leq C\|\sigma\|_{H}^{(N)} .
$$

We now use [17] as before. There is a weight function $w \in L_{1}(Q)$ with $w \geq 0$ a.e. and $\int w(x) d x=1$ so that for any $f, g \in L_{1}(3 Q)$ with $\|f\|_{L_{1}},\|g\|_{L_{1}} \leq 1$ and any measurable $E \subset Q$ we have

$$
\left(\int_{E}\left|W_{\sigma}(f, g)\right|^{r} d x\right)^{1 / r} \leq C\|\sigma\|_{H}^{(N)}\left(\int_{E} w(x) d x\right)^{1 / r-2} .
$$

Now suppose $f, g$ are supported in $Q$ and $\lambda>0$. Let $E=\{x \in Q$ : $\left|W_{\sigma}(f, g)\right|>\lambda$. Then the above equation yields

$$
\lambda|E|^{1 / r} \leq C\|\sigma\|_{H}^{(N)}\left(\int_{E} w(x) d x\right)^{1 / r-2} .
$$

On the other hand, if we apply (7.19) to $f_{t}(x)=f(x-t)$ where $t \in Q$ and note that $W_{\sigma}\left(f_{t}, g\right)=\left(W_{\sigma}(f, g)\right)_{t}$ we also obtain

$$
\lambda|E \cap(Q+t)|^{1 / r} \leq C\|\sigma\|_{H}^{(N)}\left(\int_{E} w(x-t) d x\right)^{1 / r-2} .
$$

Raising to the power $(1 / r-2)^{-1}$ and averaging gives

$$
\lambda|E|^{1 / r} \leq C\|\sigma\|_{H}^{(N)}|E|^{1 / r-2} .
$$

Thus $W_{\sigma, Q}$ maps $L_{1}(2 Q) \times L_{1}(2 Q)$ into $L_{1 / 2, \infty}(Q)$ with norm at most $C\|\sigma\|_{H}^{(N)}$.

Now let $\lambda>1$. If we define $\sigma_{\lambda}(\xi, \eta)=\sigma\left(\lambda^{-1} \xi, \lambda^{-1} \eta\right)$, then we have $\left\|\sigma_{\lambda}\right\|_{H}^{(N)}=\left\|\sigma_{\lambda}\right\|_{H}^{(N)}$ and we can apply this result to $\sigma_{\lambda}$. Notice that $W_{\sigma_{\lambda}}(f, g)(x)=W_{\sigma}\left(f_{\lambda}, g_{\lambda}\right)(\lambda x)$ where $f_{\lambda}(x)=f(\lambda x)$ and $g_{\lambda}(x)=g(\lambda x)$. This implies that for any $\lambda>0$ we have the estimate

$$
\left\|\chi_{\lambda Q} W_{\sigma}(f, g)\right\|_{L_{1 / 2, \infty}} \leq C\|\sigma\|_{H}^{(N)}\|f\|_{L_{1}}\|g\|_{L_{1}}
$$

for $f, g$ supported in $\lambda Q$. Letting $\lambda \rightarrow \infty$ gives the result.

8. Discussion on paraproducts. Paraproducts are bilinear operators of the type $\sigma_{A}$ for some specific upper (or lower) triangular matrices $A$ of zeros and ones. Paraproducts are important tools which have been used on several occasions in harmonic analysis, such as in the proof of the $T 1$ theorem of David and Journé [6]. We define the lower and upper paraproducts as the bilinear operators $\Pi_{\mathrm{L}}$ and $\Pi_{\mathrm{U}}$ with symbols

$$
\tau_{\mathrm{L}}(\xi, \eta)=\sum_{j \in \mathbb{Z}} \sum_{k \leq j-3} \widehat{\phi}_{j}(\xi) \widehat{\phi}_{k}(\eta) \quad \text { and } \quad \tau_{\mathrm{U}}(\xi, \eta)=\sum_{k \in \mathbb{Z}} \sum_{j \leq k-3} \widehat{\phi}_{j}(\xi) \widehat{\phi}_{k}(\eta)
$$


respectively. It is easy to see that $\left\|\tau_{\mathrm{L}}\right\|_{\mathcal{M}_{p_{1}, p_{2}}},\left\|\tau_{\mathrm{U}}\right\|_{\mathcal{M}_{p_{1}, p_{2}}}<\infty$ for all $0<$ $p_{1}, p_{2}<\infty$. This can be deduced in several ways, e.g. from Proposition 5.4 using Lemma 6.3 or directly from Theorem 7.2 and Proposition 3.1. We conclude that for all $0<p, q<\infty, \Pi_{\mathrm{L}}$ maps $H_{p_{1}} \times H_{p_{2}}$ to $H_{p_{0}}$ when $1 / p_{1}+1 / p_{2}=1 / p_{0}$ and to $H_{q}=L_{q}$ when $1<q<\infty$. We now turn to some endpoint cases regarding the paraproduct operator $\Pi_{\mathrm{L}}$.

Proposition 8.1. Let $0<q<\infty$. Then the paraproduct operator $\Pi_{\mathrm{L}}$ is bounded on the following products of spaces:

(1) $\mathrm{BMO} \times H_{q}\left(\mathbb{R}^{n}\right) \rightarrow H_{q}\left(\mathbb{R}^{n}\right)$, where $H_{q}=L_{q}$ when $1<q<\infty$;

(2) $\mathrm{BMO} \times H_{1}\left(\mathbb{R}^{n}\right) \rightarrow L_{1}\left(\mathbb{R}^{n}\right)$;

(3) $\mathrm{BMO} \times L_{\infty}\left(\mathbb{R}^{n}\right) \rightarrow \mathrm{BMO}$;

(4) $H_{q}\left(\mathbb{R}^{n}\right) \times L_{\infty}\left(\mathbb{R}^{n}\right) \rightarrow H_{q}\left(\mathbb{R}^{n}\right)$, where $H_{q}=L_{q}$ when $1<q<\infty$;

(5) $L_{1}\left(\mathbb{R}^{n}\right) \times L_{\infty}\left(\mathbb{R}^{n}\right) \rightarrow L_{1, \infty}\left(\mathbb{R}^{n}\right)$;

(6) $\mathrm{BMO} \times L_{1}\left(\mathbb{R}^{n}\right) \rightarrow L_{1, \infty}\left(\mathbb{R}^{n}\right)$;

(7) $L_{1}\left(\mathbb{R}^{n}\right) \times L_{1}\left(\mathbb{R}^{n}\right) \rightarrow L_{1 / 2, \infty}\left(\mathbb{R}^{n}\right)$.

Proof. Statement (1) is a classical result on paraproducts when $1<$ $q<\infty$ and we refer the reader to [19], p. 303 for a proof. Note that for a fixed $f \in \mathrm{BMO}$, the map $g \mapsto \Pi_{\mathrm{L}}(f, g)$ is a Calderón-Zygmund singular integral. The extension of (1) to $H_{q}$ for $q \leq 1$ is a consequence of the fact that if a convolution type singular integral operator maps $L_{2} \rightarrow L_{2}$ with bound a multiple of $\|f\|_{\mathrm{BMO}}$, then it also maps $H_{q}$ into itself with bound a multiple of this constant. (2) follows from a similar observation while (3) is a dual statement to (2). To prove (4) set $\widetilde{S}_{j} g=\sum_{k \leq j-3} \widetilde{\Delta}_{k} g$. We have $\Pi_{\mathrm{L}}(f, g)=\sum_{j \in \mathbb{Z}} \widetilde{\Delta}_{j} f \widetilde{S}_{j} g$ and the Fourier transform of $\widetilde{\Delta}_{j} f \widetilde{S}_{j} g$ is supported in the annulus $2^{j-2} \leq|\xi| \leq 2^{j+2}$. It follows that

$$
\left\|\Pi_{\mathrm{L}}(f, g)\right\|_{H_{q}} \leq C\left\|\left(\sum_{j \in \mathbb{Z}}\left|\widetilde{\Delta}_{j} f \widetilde{S}_{j} g\right|^{2}\right)^{1 / 2}\right\|_{H_{q}} \leq\|f\|_{H_{q}}\|M g\|_{L_{\infty}},
$$

where $M$ is the Hardy-Littlewood maximal operator, which is certainly bounded on $L_{\infty}$. To prove (5) we freeze $g$ and look at the linear operator $f \mapsto \Pi_{\mathrm{L}}(f, g)$ whose kernel is $K(x, y)=\sum_{j \in \mathbb{Z}} \phi_{j}(x-y) S_{j}(g)(x)$. It is easy to see that

$$
\left|\nabla_{y} K(x, y)\right| \leq C\|g\|_{L_{\infty}}|x-y|^{-n-1} .
$$

This estimate together with the fact that the linear operator $f \mapsto \Pi_{\mathrm{L}}(f, g)$ maps $L_{2}$ into $L_{2}$ entails that $f \mapsto \Pi_{\mathrm{L}}(f, g)$ maps $L_{1}$ into $L_{1, \infty}$ using the Calderón-Zygmund decomposition. This proves (5). To obtain (6) we use (1) (with $q=2$ ) and we apply the Calderón-Zygmund decomposition to the operator $g \mapsto \Pi_{\mathrm{L}}(f, g)$ for fixed $f \in$ BMO. Finally, (7) is a consequence of Theorem 7.6. 


\section{References}

[1] A. Bonami, Ensembles $\Lambda(p)$ dans le dual $D^{\infty}$, Ann. Inst. Fourier (Grenoble) 18 (1968), no. 2, 193-204.

[2] D. L. Burkholder, A proof of Petczyński's conjecture for the Haar system, Studia Math. 91 (1988), 79-83.

[3] R. R. Coifman, A real variable characterization of $H^{p}$, ibid. 51 (1974), 269-274.

[4] R. R. Coifman et Y. Meyer, Commutateurs d'intégrales singulières et opérateurs multilinéaires, Ann. Inst. Fourier (Grenoble) 28 (1978), no. 3, 177-202.

[5] —, 一, Au-delà des opérateurs pseudo-différentiels, Astérisque 57 (1978).

[6] G. David and J.-L. Journé, A boundedness criterion for generalized CalderónZygmund operators, Ann. of Math. 120 (1984), 371-397.

[7] C. Fefferman and E. M. Stein, $H^{p}$ spaces of several variables, Acta Math. 129 (1972), 137-193.

[8] A. M. Garsia, Martingale Inequalities, Benjamin, Reading, MA, 1973.

[9] L. Grafakos and R. Torres, Multilinear Calderón-Zygmund theory, submitted.

[10] N. J. Kalton, Plurisubharmonic functions on quasi-Banach spaces, Studia Math. 84 (1986), 297-324.

[11] C. Kenig and E. M. Stein, Multilinear estimates and fractional integration, Math. Res. Lett. 6 (1999), 1-15.

[12] M. T. Lacey and C. M. Thiele, On Calderón's conjecture, Ann. of Math. 149 (1999), 683-724.

[13] J. Lindenstrauss and L. Tzafriri, Classical Banach Spaces, I. Sequence Spaces, Ergeb. Math. Grenzgeb. 92, Springer, Berlin, 1977.

[14] -, -, Classical Banach Spaces, II. Function Spaces, Ergeb. Math. Grenzgeb. 97, Springer, Berlin, 1979.

[15] B. Muckenhoupt, On inequalities of Carleson and Hunt, in: Proc. Conf. on Harmonic Analysis in honor of Antoni Zygmund (Chicago, IL, 1981), Vol. I, Wadsworth Math. Ser., Wadsworth, Belmont, CA, 1983, 179-185.

[16] E. M. Nikishin, A resonance theorem and series in eigenfunctions of the Laplace operator, Izv. Akad. Nauk SSSR Ser. Mat. 36 (1972), 795-813.

[17] G. Pisier, Factorization of operators through $L_{p \infty}$ or $L_{p 1}$ and noncommutative generalizations, Math. Ann. 276 (1986), 105-136.

[18] E. M. Stein, Singular Integrals and Differentiability Properties of Functions, Princeton Univ. Press, Princeton, NJ, 1970.

[19] -, Harmonic Analysis: Real-Variable Methods, Orthogonality, and Oscillatory Integrals, Princeton Univ. Press, Princeton, NJ, 1993.

[20] A. Torchinsky, Real-Variable Methods in Harmonic Analysis, Academic Press, San Diego, 1986.

Department of Mathematics

University of Missouri-Columbia

Columbia, MO 65211, U.S.A.

E-mail: loukas@math.missouri.edu

nigel@math.missouri.edu 